\title{
ITÔ AND STRATONOVICH STOCHASTIC PARTIAL DIFFERENTIAL EQUATIONS: TRANSITION FROM MICROSCOPIC TO MACROSCOPIC EQUATIONS
}

\author{
BY \\ PETER M. KOTELENEZ \\ Department of Mathematics, Case Western Reserve University, 10900 Euclid Avenue, Cleveland, \\ Ohio 44106
}

\begin{abstract}
We review the derivation of stochastic ordinary and quasi-linear stochastic partial differential equations (SODE's and SPDE's) from systems of microscopic deterministic equations in space dimension $d \geq 2$ as well as the macroscopic limits of the SPDE's. The macroscopic limits are quasi-linear (deterministic) PDE's. Both noncoercive and coercive SPDE's, driven by Itô differentials with respect to correlated Brownian motions, are considered. For the solutions of semi-linear noncoercive SPDE's with smooth and homogeneous diffusion kernels we show that these solutions can be obtained as solutions of first-order SPDE's, driven by Stratonovich differentials and their macroscopic limit, and are solutions of a class of semi-linear second-order parabolic PDE's. Further, the space-time covariance structure of correlated Brownian motions is described and for space dimension $d \geq 2$ the long-time behavior of the separation of two uncorrelated Brownian motions is shown to be similar to the independent case.
\end{abstract}

1. From microscopic to mesoscopic and macroscopic equations. Einstein [6] develops a model of Brownian motions to describe the displacement of large solute particles as the result of the interaction (collisions) with the small solvent particles. Einstein assumes that the motions of the large particles are statistically independent, provided they are sufficiently far separated. In the rigorous mathematical framework of several Brownian motions (or "Wiener processes") the assumption of independence, irrespective of the distance, has become a widely accepted model in stochastic analysis and the associated parabolic partial differential equations. In applications, however, it is well known that at short distances the depletion effect generates an attractive force between the large particles (cf. Kotelenez, Leitman and Mann 22] and the references therein), where depletion refers to the fact that the number of small particles per small volume between two large particles drops if the distance between the two large particles becomes small.

Received May 15, 2007.

2000 Mathematics Subject Classification. Primary 60H10, 60H05, 60H30, 60F17.

E-mail address: pxk4@po.cwru.edu

(C)2008 Brown University

Reverts to public domain 28 years from publication 
In particular, if the distance between the two large particles is less than a critical positive parameter, $\sqrt{\varepsilon}$, then the surrounding medium of small particles can no longer be homogeneous and isotropic and the two large particles must become statistically correlated. $\sqrt{\varepsilon}$ will be called the "correlation length".

Traditionally, there are three levels to describe the time evolution of particle distributions: microscopic, mesoscopic and macroscopic (cf., e.g., Haken [13] or van Kampen [16]).

On a microscopic level, Newtonian mechanics governs the equations of motion of the individual atoms or molecules 1 For our system of large (solute) and small (solvent) particles we can describe the model as follows: Suppose there are $N$ large particles and infinitely many small particles distributed in the Euclidean state space $\mathbf{R}^{d} 2$ The position of the (center of the) $i$ th large particle at time $t$ will be denoted $r^{i}(t)$ and its velocity $v^{i}(t)$. The corresponding position of the (center of the) $\lambda$ th small particle and its velocity will be denoted $q^{\lambda}(t)$ and $w^{\lambda}(t)$, respectively. $\tilde{m}$ is the mass of a small particle and $m$ the mass of a large particle. The empirical distributions of large and small particles are (formally) given by

$$
\mathcal{X}_{N}(d r, t):=m \sum_{j=1}^{N} \delta_{r^{j}(t)}(d r), \quad \mathcal{Y}(d q, t):=\tilde{m} \sum_{\lambda} \delta_{q^{\lambda}(t)}(d q) .
$$

1a) Transition from microscopic to mesoscopic equations. To simplify the transition from microscopic to mesoscopic a mean-field interaction between large and small particles is assumed, where spatially extended particles are replaced by point particles and "large" and "small" refer to the mass of a particle. Further, the interaction between small particles is assumed to be negligible, and the interaction between large particles can be (temporarily) neglected 3 Denote the potential governing the interaction between large and small particles by $U_{\varepsilon}(\mu, r-q)$ on a space-time continuum, and let $\mu>0$ be a friction coefficient for the large particles (cf. the following (1.1)). Setting $G_{\varepsilon}(\mu, r):=-\nabla U_{\varepsilon}(\mu, r)$, the interaction between small and large particles can be described by the following infinite system of nonlinear coupled oscillators:

$$
\left.\begin{array}{c}
\frac{d}{d t} r^{i}(t)=v^{i}(t), \quad r^{i}(0)=r_{0}^{i}, \\
\frac{d}{d t} v^{i}(t)=-\mu v^{i}(t)+\frac{1}{m} \int G_{\varepsilon}\left(\mu, r^{i}(t)-q\right) \mathcal{Y}(d q, t), \quad v^{i}(0)=v_{0}^{i}, \\
\frac{d}{d t} q^{\lambda}(t)=w^{\lambda}(t), \quad q^{\lambda}(0)=q_{0}^{\lambda}, \\
\frac{d}{d t} w^{\lambda}(t)=\frac{1}{m} \int G_{\varepsilon}\left(\mu, q^{\lambda}(t)-r\right) \mathcal{X}_{N}(d r, t), \quad w^{\lambda}(0)=w_{0}^{\lambda} .
\end{array}\right\}
$$

Here and in what follows, the integration domain will be over all of $\mathbf{R}^{d}$ if no integration domain is specified. Observe that $G_{\varepsilon}(\mu, r-q)$ has the dimensions $\frac{\ell}{T^{2}}$ (length over time squared).

On the "mesoscopic" level the motion of the large particles is stochastic and the randomness of the motion is determined by the surrounding medium, which is obtained from

\footnotetext{
${ }^{1}$ I.e., we restrict ourselves to classical physics.

${ }^{2}$ Infinitely many small particles are needed to generate independent increments in the limiting Brownian motion. Cf. our Comment, Part (i) at the end of this section and Kotelenez [19].

${ }^{3}$ As the interaction between large particles occurs on a much slower time scale than the interaction between large and small particles, it can be included after the mesoscopic scaling limit, employing fractional steps (cf. Goncharuk and Kotelenez [12]).
} 
the system of small particles. Observe that for only one large particle the surrounding medium can be assumed to be homogeneous and isotropic. Therefore, given the preceding simplifying assumptions, the marginal motion of any given large particle must be Brownian (a Wiener process), and if large particles are sufficiently separated (dilute system), their motions should be approximately independent. However, if the distance between two large particles becomes smaller than (a multiple of) the correlation length $\sqrt{\varepsilon}$, then the particles need to be correlated and have a tendency to attract one another. We will call such a system of $N$ large particles $N$ "correlated Brownian motions" (cf. also Definition 3.2). Following Kotelenez [18]-[20, a model of correlated Brownian motions can be defined as follows: Let $w(d q, d s)$ be the standard Gaussian white noise on $\mathbf{R}^{d} \times \mathbf{R}_{+}$ (which is a space-time generalization of the time increments of a standard scalar-valued Brownian motion, $\beta(d s))$ 4 $w(d q, d s)$ is defined on the same probability space as the initial positions $\left(r^{1}(0), \ldots, r^{N}(0)\right)$ and assumed to be independent of the initial positions. Assume for the kernel that

$$
G_{\varepsilon}(r) \approx \frac{1}{\mu} G_{\varepsilon}(\mu, r), \text { as } \mu \longrightarrow \infty .
$$

Then the positions of the large particles are the solutions of the stochastic integral equations:

$$
r^{i}(t)=r^{i}(0)+\int_{0}^{t} \int G_{\varepsilon}\left(r^{i}(u)-q\right) w(d q, d u), \quad i=1, \ldots, N .
$$

The equations (1.2) are coupled only through the Gaussian space-time white noise $w(d q, d u)$, which has the units of time. For a large class of kernels $G_{\varepsilon},(1.2)$ has a unique solution and the $d$-dimensional components are square integrable continuous martingales. Moreover, for each $i=1, \ldots, N, r^{i}(\cdot)-r^{i}(0)$ are Brownian motions (cf. Section 3 for more details and an analysis of the correlations between the motions of two different particles).

Next, we give sufficient conditions for the transition from the microscopic description (1.1) to the mesoscopic (i.e., stochastic) description (1.2):

- Formation of small clusters (ensembles) of particles if their initial positions and velocities are similar (coarse graining in space) 5

- Replace the time derivative in (1.1) by an Euler scheme (coarse graining in time).

- Randomization of the initial distribution of clusters, where the probability distribution is determined by the relative sizes of the clusters. Assume statistical independence of initial distributions of different clusters.

- The initial average velocity, $\left\langle w_{0}\right\rangle$, of the small particles and the friction coefficient $\mu$ for the large particles tend to infinity such that $\sqrt{\varepsilon} \mu \ll\left\langle w_{0}\right\rangle$.

\footnotetext{
${ }^{4}$ If $A$ is a Borel subset of $[0, \infty)$ of finite Lebesgue measure $|A|, \int_{A} \beta(d t)$ is normally distributed with mean 0 and variance $|A|$ and if $A_{1} \cap A_{2}=\emptyset$ for two Borel subsets of $[0, \infty)$ of finite Lebesgue measure, then $\int_{A_{1}} \beta(d t)$ and $\int_{A_{2}} \beta(d t)$ are independent. Similarly, let $B, B_{1}, B_{2}$ be Borel subsets of $\mathbf{R}^{d}$ of finite Lebesgue measure. Then $A \times B$ is a Borel set of finite Lebesgue measures. Then $\int_{A} \int_{B} w(d q, d t)$ is normally distributed with mean 0 and variance $|A| \cdot|B|$ and if $A_{1} \times B_{1} \cap A_{2} \times B_{2}=\emptyset$, then $\int_{A_{1}} \int_{B_{1}} w(d q, d t)$ and $\int_{A_{2}} \int_{B_{2}} w(d q, d t)$ are independent (cf. Walsh [37).

${ }^{5}$ In a spatially coarse grained model, particles whose initial positions and velocities are almost identical are considered a cluster and are treated as one small particle.
} 
- Allow the small particles to escape to infinity after having interacted with the large particles for a macroscopically small time, assuming that the space dimension is $d \geq 26$

Under these assumption, Kotelenez [19] (in addition to a suitable convergence of the initial conditions) derives a "mesoscopic" limit theorem in which the positions of the large particles in a sequence of coarse grained versions of (1.1) tend to the solutions of (1.2) weakly in the Skorohod space of cadlag functions with values in $\mathbf{R}^{d N}$ for $d \geq 2$.

Comments.

(i) The escape to infinity after a short period of interaction with the large particles is necessary to generate independent increments in the limit. This can be seen as follows. The small time steps induce a partition of the time axis into small time intervals. Further, denote the domain of concentration of the large particles by $\mathcal{D}$. Since the number of large particles is finite, $\mathcal{D}$ may be assumed to be bounded and expands slowly due to the slow motion of the large particles. In each of the small time intervals the large particles are being displaced by the interaction with clusters of small particles being in $\mathcal{D}$ during that small time interval. Note that the vast majority of small particles had previously not interacted with the large particles prior to entering $\mathcal{D}$. After that time step most small particles leave $\mathcal{D}$ and outside $\mathcal{D}$ their velocities remain unchanged. Hence, they escape to infinity, i.e. their distance to $\mathcal{D}$ tends to infinity. This is true for every small time interval. Since clusters have started independently, this implies almost independence of the displacements of the large particles in different time intervals. In a scaling limit, as the initial velocities of the small particles and the friction coefficient for the large particles tend to infinity, the motions of the large particles have orthogonal increments 7 It is the orthogonal increments in time, where an infinite system of small particles and the escape to infinity are needed. We can obtain a similar result if there is no friction in the equations for the large particles. In this case the limit would be an OrnsteinUhlenbeck model (described by stochastic second-order equations), where the velocities perform correlated Brownian motions. For the case of one large particle we refer to results obtained by Dürr, Goldstein and Lebowitz [4, Sinai and Soloveichik [33], Szász and Tóth 34 and the references therein.

(ii) Suppose that small particles move with different velocities. If most of the small particles, moving in the direction of a large particle, can avoid collisions with other small particles (as, e.g., in a rare gas or in the PHS model), fast small particles coming from "far away" can collide with a given large particle at approximately the same time as slow small particles which were close to the large particle before the collision. If, in repeated microscopic time steps, collisions of a given small particle with the same large particle are negligible, then in a mesoscopic time unit $\delta \sigma$, the collision dynamics can be replaced by long-range mean field dynamics. Dealing with a wide range of velocities, as in the

\footnotetext{
${ }^{6}$ This hypothesis seems to be acceptable if for spatially extended particles the interparticle distance is considerably greater than the diameter of a typical particle. The assumption holds for a gas (cf. Lifshits and Pitayevskii [28], Ch.1, 33 ), but not for a liquid, like water. For a liquid, we refer to the PHS model, reviewed in Section 2 of Kotelenez, Leitman and Mann (loc. cit.).

${ }^{7}$ The marginal motion of each large particle has independent increments. But the mean field interaction with the medium of small particles correlates their motions, which leads to a weaker property of orthogonal increments of the system of large particles.
} 
Maxwellian case, and working with discrete time steps, a long range force is generated. If we assume that the empirical velocity distribution of the small particles is approximately Maxwellian, the aforementioned mean field force can be given by the following expression (cf. Kotelenez [21], (1.2)):

$$
G_{\varepsilon, M}(\mu, R-q) \approx c_{\varepsilon}(R-q) \mu e^{-\frac{|R-q|^{2}}{2 \varepsilon}} .
$$

Here, $\mu$ is the friction coefficient for the large particles and $c_{\varepsilon}$ is a positive constant with the unit $\frac{1}{T} . c_{\varepsilon}$ has to be defined such that, choosing

$$
G_{\varepsilon, M}(r):=c_{\varepsilon} r e^{-\frac{|r|^{2}}{2 \varepsilon}}
$$

in the kinetic stochastic equation (1.2), $\left|G_{\varepsilon, M}(r)\right|^{2}$ must approximate the $\delta$-function, as $\varepsilon \downarrow 0$, where $|\cdot|$ denotes the Euclidean norm in $\mathbf{R}^{d}$.

Obviously, this example can be immediately generalized to an arbitrary velocity field of the small particles. A more realistic model would be some (possibly nonlinear) transformation of the velocity field, taking into account collisions between small particles, etc. For the purpose of our paper, it suffices to work with a general mean-field force kernel $G_{\varepsilon}$ as in (1.2) and show that for certain kernels the right-hand side of (1.2) behaves according to our requirements, stated at the beginning of this section.

As a result of the mesoscopic limit theorem, the small particles become a Brownian random medium which drives the motion of the $N$ large point particles in $\mathbf{R}^{d}, d \geq$ 2. In what follows we will drop the epithet "large" and study the motion of the $N$ particle system under the influence of additional forces. As in (1.2), the position of the $i$-th particle at time $t$ will be denoted $r^{i}(t)$ and its mass $m_{i}$. Then the empirical mass distribution (also called the "empirical process") at time $t$ is given by $\mathcal{X}_{N}(t):=$ $\sum_{i=1}^{N} m_{i} \delta_{r^{i}(t)}$, where $\delta_{r}$ is the point measure concentrated in $r$. To make the arguments more transparent we will choose $m_{i}=\frac{1}{N}$ for $i=1, . ., N$ in what follows.

For the "macroscopic" description we refer to the following (1.7) and the transition from mesoscopic to macroscopic in Theorem 1.3.

1b) Transition from mesoscopic to macroscopic equations. $\left(\Omega, \mathcal{F}, \mathcal{F}_{t}, P\right)$ is a stochastic basis with right continuous filtration. All our stochastic processes are assumed to live on $\Omega$ and to be $\mathcal{F}_{t}$-adapted (including all initial conditions in SODE's and SPDE's). The stochastic component of the displacement of $r^{i}(t)$ in a short time increment should be Brownian (multiplied by some diffusion coefficient, which may depend both on $r^{i}(t)$ and on $\mathcal{X}_{N}(t)$ ). Before precisely formulating the appropriate generalization of (1.2), we first need to comment on a more traditional perturbation of the position of the $i$-th particle by Brownian noise.

(I) Denote the space of Borel probability measures on $\mathbf{R}^{d}$ by $\mathbf{M}_{1}$, endowed with a suitable Wasserstein metric (cf. (2.2)). $F_{0}(r, \mu, t)$ is a "nice" $\mathbf{R}^{d}$-valued function on $\mathbf{R}^{d} \times$ $\mathbf{M}_{1} \times[0, \infty)$, jointly measurable in all arguments and where "nice" in this introduction will refer to appropriate Lipschitz and linear growth assumptions. The subscript 0 indicates that there is no correlation between the Brownian noises for different particles. $\mathcal{M}_{d \times d}$ denotes the $d \times d$ matrices over $\mathbf{R}$. Further, let $\mathcal{J}_{0}(r, \mu, t)$ be a "nice" $\mathcal{M}_{d \times d}$-valued function, depending on the positions of a particle, the empirical distribution $\mathcal{X}_{0, N}(t)$ with weights $m_{i}:=\frac{1}{N}$ and time $t$. Finally, $\left\{\beta^{i}(\cdot)\right\}$ is a system of i.i.d. $\mathbf{R}^{d}$-valued standard 
Brownian motions. Consider the stochastic ordinary differential equations (SODE's) in the sense of Itô for the displacement of $r^{i}$ of the following type (cf., e.g., Oelschläger [30] and Gärtner [9]):

$$
\left.\begin{array}{l}
d r_{0, N}^{i}(t)=F_{0}\left(r_{0, N}^{i}(t), \mathcal{X}_{0, N}(t), t\right) d t+\mathcal{J}_{0}\left(r_{0, N}^{i}(t), \mathcal{X}_{0, N}(t), t\right) d \beta^{i}(t), \\
r_{0, N}^{i}(s)=q_{0}^{i}, i=1, \ldots, N, \quad \mathcal{X}_{0, N}(t):=\sum_{i=1}^{N} \frac{1}{N} \delta_{r_{0, N}^{i}}(t) .
\end{array}\right\}
$$

We next give an intuitive argument why the empirical processes in (1.4) converge to a macroscopic (i.e., deterministic) quantity. Let $\mathbf{0} \in \mathcal{M}_{d \times d}$ be the matrix with all entries being equal to 0 . The two-particle diffusion matrix of the noise is given by

$$
\tilde{D}_{0}\left(r^{i}, r^{j}, \mu, t\right):=\left\{\begin{array}{lll}
\mathcal{J}_{0}\left(r^{i}, \mu, t\right) \mathcal{J}_{0}^{T}\left(r^{j}, \mu, t\right), & \text { if } & i=j, \\
\mathbf{0}, & \text { if } & i \neq j,
\end{array}\right.
$$

where " $A B$ " denotes the matrix multiplication of matrices $A$ and $B$ and $B^{T}$ is the transpose of a matrix $B$. Further, " $A_{k \ell}$ " will denote the entries of the matrix $A$. Choose a twice continuously differentiable function $\varphi$ on $\mathbf{R}^{d}$ and denote by $\langle\cdot, \cdot\rangle$ the duality between measures and continuous functions. Under nontrivial assumptions on the coefficients in (1.4), Itô's formula yields the following incremental quadratic variations for the empirical process associated with (1.4):

$$
\left.\begin{array}{rl}
d\left[\left\langle\mathcal{X}_{0, N}(t), \varphi\right\rangle\right]= & \sum_{i=1}^{N} \frac{1}{N^{2}} \sum_{k, \ell=1}^{d}\left(\partial_{k} \varphi\right)\left(r_{0, N}^{i}(t)\right)\left(\partial_{\ell} \varphi\right)\left(r_{0, N}^{i}(t)\right) \\
& \times \tilde{D}_{0, k, \ell}\left(r_{0, N}^{i}(t), r_{0, N}^{i}(t), \mathcal{X}_{0, N}(t), t\right) d t \\
= & O_{\varphi}\left(\frac{1}{N}\right) d t
\end{array}\right\}
$$

since we have $N$ terms in the sum (1.6), divided by $N^{2}$. Here and in what follows, $\partial_{k}, \partial_{k, \ell}^{2}$ denote the first and second partial derivatives with respect to the coordinates of $r$. Employing Doob's inequality, we obtain from (1.6) that the noise must converge to 0, as $N \rightarrow \infty$. Set $D(r, \mu, t):=\tilde{D}(r, r, \mu, t)$ and let " $\Longrightarrow$ " denote weak convergence on some topological space and "•" the scalar product on $\mathbf{R}^{d}$. One can show that $X_{0, N}(\cdot) \Longrightarrow$ $X_{0, \infty}$, as $N \longrightarrow \infty$, where now $X_{0, \infty}$ is the solution of the following quasi-linear parabolic partial differential equation (PDE) of McKean-Vlasov type (cf. Oelschläger (loc. cit.) and Gärtner (loc. cit.)):

$$
\left.\begin{array}{rl}
\frac{\partial}{\partial t} X_{0, \infty} & =\frac{1}{2} \sum_{k, \ell=1}^{d} \partial_{k \ell}^{2}\left(D_{0, k \ell}\left(\cdot, X_{0, \infty}, t\right) X_{0, \infty}\right)-\nabla \bullet\left(X_{0, \infty} F_{0}\left(\cdot, X_{0, \infty}, t\right)\right), \\
X_{0, \infty}(0) & =\mu
\end{array}\right\}
$$

(II) The generalization of (1.2) was analyzed by Kotelenez [17]-[18. Take i.i.d. Gaussian standard white noise random fields $w_{\ell}(d q, d t)$ on $\mathbf{R}^{d} \times \mathbf{R}_{+}, \ell=1, \ldots, d$, as a stochastic perturbation for the positions of the particles. Further, let $\mathcal{J}_{\varepsilon}(r, q, \mu, t)$ be a "nice" $\mathcal{M}_{d \times d}$-valued function, jointly measurable in all arguments, depending on the position of the particle, the spatial noise coordinate, the empirical distribution, time $t$ and a correlation parameter $\varepsilon>0$ (cf., e.g., (1.3)). In addition to Lipschitz and measurability assumptions, "nice" means here that the one-dimensional components of $\mathcal{J}_{\varepsilon}(r, q, \ldots, t)$ have to be square integrable in $p$ with respect to the Lebesgue measure $d p . F_{\varepsilon}$ is as in 
(1.4). Consider the following system of Itô-SODE's driven by $w(d r, d t) 8$

$$
\left.\begin{array}{rl}
r_{\varepsilon, N}^{i}= & F_{\varepsilon}\left(r_{\varepsilon, N}^{i}(t), \mathcal{X}_{\varepsilon, N}(t), t\right) d t+\int \mathcal{J}_{\varepsilon}\left(r_{\varepsilon, N}^{i}(t), q, \mathcal{X}_{\varepsilon, N}(t), t\right) w(d q, d t), \\
r_{\varepsilon, N}^{i}(0)= & q_{\varepsilon}^{i}, i=1, \ldots, N, \quad \mathcal{X}_{\varepsilon, N}(t):=\sum_{i=1}^{N} \frac{1}{N} \delta_{r_{\varepsilon, N}^{i}}(t) .
\end{array}\right\}
$$

Under appropriate Lipschitz conditions (cf. (2.4)) Kotelenez [18] shows that (1.11) has a unique strong Itô solution which is an $\mathbf{R}^{d N}$-valued diffusion process.

Adjusting the notation of approach (I) to the setting of (1.8), we obtain the twoparticle diffusion matrix:

$$
\tilde{D}_{\varepsilon}\left(r^{i}, r^{j}, \mu, t\right):=\int \mathcal{J}_{\varepsilon}\left(r_{\varepsilon}^{i}, q, \mu, t\right) \mathcal{J}_{\varepsilon}^{T}\left(r_{\varepsilon}^{j}, q, \mu, t\right) d q \quad \forall i, j=1, \ldots, N .
$$

Differently from (1.5), the pair correlations do not disappear for $i \neq j$. We obtain for the empirical process $\mathcal{X}_{\varepsilon, N}(t)$ from (1.9) the following incremental quadratic variations:

$$
\left.\begin{array}{rl}
d\left[\left\langle\mathcal{X}_{\varepsilon, N}(t), \varphi\right\rangle\right]= & \sum_{i, j=1}^{N} \frac{1}{N^{2}} \sum_{k, \ell=1}^{d}\left(\partial_{k} \varphi\right)\left(r_{\varepsilon, N}^{i}(t)\right)\left(\partial_{\ell} \varphi\right)\left(r_{\varepsilon, N}^{i}(t)\right) \\
& \times \tilde{D}_{\varepsilon, k, \ell}\left(r_{\varepsilon, N}^{i}(t), r_{\varepsilon, N}^{j}(t), \mathcal{X}_{\varepsilon, N}(t), t\right) d t \\
= & O_{\varphi}(1) d t
\end{array}\right\}
$$

because we now have $N^{2}$ terms in the sum, divided by $N^{2}$. Consequently, the noise does not disappear in the limit as $N \rightarrow \infty$. To better understand the limit, let us first derive an equation for the empirical process. Choose a smooth test function $\varphi$. Applying Itô's formula to $\varphi\left(r_{N}^{i}(t)\right)$ and integrating by parts in the generalized sense yields

$$
\left.\begin{array}{l}
\left\langle d \mathcal{X}_{\varepsilon, N}(t), \varphi\right\rangle=\left\langle\frac{1}{2} \sum_{k, \ell=1}^{d} \partial_{k \ell}^{2}\left(D_{\varepsilon, k \ell}\left(\cdot, \mathcal{X}_{\varepsilon, N}, \cdot, t\right) \mathcal{X}_{\varepsilon, N}(t)\right) d t, \varphi\right\rangle \\
-\left\langle\nabla \bullet\left(\mathcal{X}_{N}(t) F_{\varepsilon}\left(\cdot, \mathcal{X}_{\varepsilon, N}, t\right)\right) d t, \varphi\right\rangle \\
-\left\langle\nabla \bullet\left(\mathcal{X}_{N}(t) \int \mathcal{J}\left(\cdot, p, \mathcal{X}_{\varepsilon, N}, t\right) w(d p, d t)\right), \varphi\right\rangle,
\end{array}\right\}
$$

where we abbreviated

$$
D_{\varepsilon}(r, \mu, t):=\tilde{D}_{\varepsilon}(r, r, \mu, t) .
$$

Hence, the empirical process itself is a weak solution (in the sense of PDE's) of the following quasi-linear stochastic partial differential equation (SPDE) (1.13), where the derivatives are taken in the distributional sense. In what follows a "solution" of an SPDE is by definition a weak solution in the sense of PDE's but strong in the sense of stochastic analysis; i.e., all quantities are defined on the same probability space with the same noise, etc. Assuming global Lipschitz assumptions on the coefficients in the variables $(r, \mu)$, Kotelenez [18] shows that we can pass to the limit, as $N \rightarrow \infty$, provided that the initial conditions $\mathcal{X}_{\varepsilon, N}$ converge suitably to a measure-valued initial condition $\mathcal{X}_{\varepsilon, 0}$. As convergence in the Wasserstein metric is stronger than weak* convergence with respect to smooth test functions, we obtain that the limit is also a solution of (1.13).

\footnotetext{
8 The mean field kernel $G_{\varepsilon}$ from (1.2) describes only the interaction between small and large particles. On the coarser mesoscopic time scale we include also interaction between large particles and the possible dependence of the stochastic term on the mass distribution of the large particles, leading to $F$ and the more general kernel $\mathcal{J}_{\varepsilon}$. A precise mathematical way of doing this is the method of fractional steps. Cf. Goncharuk and Kotelenez 12 .
} 
(For more details cf. the following Section 4.)

$$
\left.\begin{array}{rl}
d \mathcal{X}_{\varepsilon}= & \left\{\frac{1}{2} \sum_{k, \ell=1}^{d} \partial_{k \ell}^{2}\left(D_{\varepsilon, k \ell}\left(\cdot, \mathcal{X}_{\varepsilon}, t\right) \mathcal{X}_{\varepsilon}\right)-\nabla \bullet\left(\mathcal{X}_{\varepsilon} F_{\varepsilon}\left(\cdot, \mathcal{X}_{\varepsilon}, t\right)\right)\right\} d t \\
& -\nabla \bullet\left(\mathcal{X}_{\varepsilon} \int \mathcal{J}_{\varepsilon}\left(\cdot, p, \mathcal{X}_{\varepsilon}, t\right) w(d p, d t)\right), \\
\mathcal{X}_{\varepsilon}(0)= & \mathcal{X}_{\varepsilon, 0} .
\end{array}\right\}
$$

REMARK 1.1. (i) For smooth (i.e., density-valued) initial conditions and smooth coefficients, Kotelenez [18, obtains smooth solutions of (1.13). Under appropriate assumptions on the initial conditions and the coefficients, Kotelenez and Kurtz [23] show that for $d \geq 2$ the solutions of (1.13) converge weakly to the solution of (1.7), as $\varepsilon \rightarrow 0$; i.e., the solutions of (1.13) become macroscopic in the limit as the correlation length tends to 0 .

(ii) Let us call the limit $N \rightarrow \infty$ in both (I) and (II) a "continuum limit" because most of the time this limit leads to a continuous mass distribution. Accordingly, in approach (I) the continuum limit and the macroscopic limit are performed simultaneously. In contrast, the particle approach taken in (II) performs first the continuum limit and then the macroscopic limit. The advantage of (II) is the mesoscopic equation (1.13) on a continuum, whose stochastic term is small for small correlation length $\sqrt{\varepsilon}$. Note that the correlation length incorporates into the mesoscopic continuum model depletion and other effects of the underlying discrete microscopic model (cf. our Section 3 for qualitative results).

Next we comment on models related to (II).

Borkar [1] uses a Gaussian random field, called a "Brownian medium", as a driving term for stochastic ordinary differential equations (SODE's). Kunita's approach (26] and the references therein) is similar, but he goes beyond Borkar's work by considering flows of SODE's and bilinear SPDE's generated by those flows. The coefficients in Kunita's approach do not depend on the empirical distribution. Moreover, Kotelenez and Kurtz (loc. cit.) show that Kunita's space-time Gaussian random field can be represented by the convolution of a diffusion kernel (similar to $G_{\varepsilon}$ in (1.2)) with the space-time field $w(d q, d t)$.

For approaches similar to (II), where the coefficients depend on the empirical distribution, we first derive an alternative formulation of the noise in (1.8). Let $\mathbf{H}_{0}$ be the space of measurable functions on $\mathbf{R}^{d}$ which are square integrable with respect to the Lebesgue measure and let $|\cdot|_{0}$ be the usual $L_{2}$-norm, which is induced by the scalar product

$$
\langle f, g\rangle_{0}:=\int f(q) g(q) d q
$$

for $f, g \in \mathbf{H}_{0}$. Let $\left\{\phi_{n}\right\}_{n \in N}$ be a complete orthonormal system (CONS) in $\mathbf{H}_{0}$ and define an $\mathcal{M}_{d \times d}$-valued function $\widehat{\phi}_{n}$ whose entries on the main diagonal are all $\widehat{\phi}_{n}$ and whose other entries are all 0 . Set

$$
\beta^{n}(t):=\int_{0}^{t} \int \widehat{\phi}_{n}(p) w(d p, d s)
$$

Then the $\beta^{n}(\cdot)$ are i.i.d. standard $\mathbf{R}^{d}$-valued Wiener processes. Moreover, for any continuous, adapted square integrable process $z(\cdot)$ and continuous adapted $\mathbf{M}_{1}$-valued 
measure process $\tilde{\mathcal{Y}}$

$$
\int \mathcal{J}(z(t), p, \tilde{\mathcal{Y}}(t), t) w(d p, d t)=\sum_{n=1}^{\infty} \int \mathcal{J}(z(t), p, \tilde{\mathcal{Y}}(t), t) \widehat{\phi}_{n}(p) d p d \beta^{n}(t)
$$

(cf. Jetschke [15 and Kotelenez [17-[18]). Abbreviating

$$
\sigma_{\varepsilon, n}(r, \mu, t):=\int \mathcal{J}_{\varepsilon}(r, q, \mu, t) \hat{\phi}_{n}(q) d q,
$$

(1.8) becomes equivalent to the following $N$-system of Itô SODE, driven by infinitely many i.i.d. Brownian motions:

$$
\left.\begin{array}{rl}
d r_{\varepsilon, N}^{i} & =F_{\varepsilon}\left(r_{\varepsilon, N}^{i}(t), \mathcal{X}_{\varepsilon, N}(t), t\right) d t+\sum_{n=1}^{\infty} \sigma_{\varepsilon, n}\left(r_{\varepsilon, N}^{i}(t), \mathcal{X}_{\varepsilon, N}(t), t\right) d \beta^{n}(t), \\
r_{\varepsilon, N}^{i}(0) & =q_{\varepsilon}^{i}, i=1, \ldots, N, \quad \mathcal{X}_{\varepsilon, N}(t):=\sum_{i=1}^{N} \frac{1}{N} \delta_{r_{\varepsilon, N}^{i}}(t) .
\end{array}\right\}
$$

Vaillancourt [36] essentially considers stochastic ordinary differential equations (SODE's) of type (1.18) where only the first $N$ Brownian motions are used for the perturbation of $N$ particles. As $N \rightarrow \infty$, the empirical process converges to an SPDE solution of a martingale problem. Choosing our kernel $\mathcal{J}_{\varepsilon}$ such that the Fourier expansion $(1.16) /(1.17)$ yields only $N$ terms we obtain for each $N$ a system equivalent to the case in Vaillancourt. (Cf. also Dawson, Vaillancourt and Wang [2.)

Recall that (1.13) was derived through the Itô formula (i.e., the extended chain rule), applied to $\varphi\left(r_{N}^{i}(t)\right)$, where $\varphi$ was a smooth test function and $r_{N}^{i}(t)$ the position of the $i$-th particle, described by the SODE's (1.8). This procedure begs the question whether a change of (1.8) into Stratonovich form would yield a first-order SPDE as a result of the usual chain rule for $\varphi\left(r_{N}^{i}(t)\right)$. Typically the Stratonovich integral requires the integrands to be semi-martingales; i.e., it requires more regularity than the Itô integral (cf., e.g., Ikeda and Watanabe [14]). If $\mathcal{J}_{\varepsilon}(r, \mathcal{X}(t), t)$ were a semi-martingale we could derive the correction term through the Itô-Wentzell formula (cf. Rozovsky [32], Ch. 1, Theorem 9 or Kunita [26, Section 3.3). However, due to the dependence on the measure process, it is not easy to give nontrivial conditions for $\mathcal{J}_{\varepsilon}(r, \mathcal{X}(t), t)$ to be a semi-martingale (cf. Kotelenez [21], Section 15, for some partial results). Therefore, we now consider a special case of the system of SODE's (1.8), where the diffusion kernel does not depend on a measure process:

$$
\left.\begin{array}{cl}
d r_{\varepsilon, N}^{i}= & F_{\varepsilon}\left(r_{\varepsilon, N}^{i}(t), \mathcal{X}_{\varepsilon, N}(t), t\right) d t+\int \mathcal{J}_{\varepsilon}\left(r_{\varepsilon, N}^{i}(t), q, t\right) w(d q, d t), \\
r_{\varepsilon, N}^{i}(0)= & q_{\varepsilon}^{i}, i=1, \ldots, N, \quad \mathcal{X}_{\varepsilon, N}(t):=\sum_{i=1}^{N} \frac{1}{N} \delta_{r_{\varepsilon, N}^{i}}(t) .
\end{array}\right\}
$$

Based on Itô's formula, the empirical process and the limiting measure process give a PDE-weak solution of the semi-linear SPDE for the mass distribution (cf. (1.13)):

$$
\left.\begin{array}{rl}
d \mathcal{X}_{\varepsilon}= & \left\{\frac{1}{2} \sum_{k, \ell=1}^{d} \partial_{k \ell}^{2}\left(D_{\varepsilon, k \ell}(\cdot, t) \mathcal{X}_{\varepsilon}\right)-\nabla \bullet\left(\mathcal{X}_{\varepsilon} F_{\varepsilon}\left(\cdot, \mathcal{X}_{\varepsilon}, t\right)\right)\right\} d t \\
& -\nabla \bullet\left(\mathcal{X}_{\varepsilon} \int \mathcal{J}_{\varepsilon}\left(\cdot, p, \mathcal{X}_{\varepsilon}, t\right) w(d p, d t)\right), \\
\mathcal{X}_{\varepsilon}(0)= & \mathcal{X}_{\varepsilon, 0} .
\end{array}\right\}
$$

Assume additional smoothness of the diffusion kernel $\mathcal{J}_{\varepsilon}$ and that the diffusion coefficients are homogeneous $((4.5))$ and that the divergence of $\tilde{D}$ at $(0, t)$ equals 0 for all $t$ $((5.7))$. Under these assumptions we obtain in Section 5, Theorem 5.3, a Stratonovich 
representation for (1.19) 9 whence by the usual chain rule the empirical process and its measure limit, satisfying the second-order Itô SPDE (1.20), are solutions of the first-order Stratonovich SPDE:

$$
\begin{aligned}
& d \mathcal{X}_{\varepsilon}=-\nabla \bullet\left(\mathcal{X}_{\varepsilon}\left(\cdot, \mathcal{X}_{\varepsilon}, t\right)\right) d t-\nabla \bullet\left(\mathcal{X}_{\varepsilon} \int \mathcal{J}_{\varepsilon}(\cdot, p, t)\right) w(d p, \circ d t), \\
& \mathcal{X}(0)=\mathcal{X}_{0}
\end{aligned}
$$

Here "o" denotes the Stratonovich differential whose definition for Brownian motions can be extended to $w(d p$, odt) through the Fourier expansion (1.16) (cf. (5.6)).

We assume in what follows that (1.7) is itself semi-linear with $\mathcal{J}_{0}$ independent of $r$ and $\mu$ and that (4.5), (5.5) and (5.7) hold. The Kotelenez-Kurtz assumptions on the special case of (1.20), with $\sigma_{\varepsilon}(t)$ the nonnegative square root of $D_{\varepsilon}(0, t)$, are as follows (where we refer to Kotelenez and Kurtz (loc. cit.) for examples):

\section{Hypothesis 1.2.}

(i) Suppose that for each $t \geq 0, \sigma_{\varepsilon}(t)$ is invertible and, for $d=2, \sigma_{\varepsilon}(t)$ is twice continuously differentiable.

(ii) Suppose that for any compact subset $\mathcal{K}$ of $\mathbf{R}^{d}$, any compact subset $\mathcal{C} \subset \mathbf{M}_{1}$, any $T:>0$ and any $\delta>0$ the following holds 10

$$
\begin{aligned}
& \limsup _{\varepsilon \downarrow 0} \sup _{r \in \mathcal{K}} \sup _{0 \leq t \leq T}\left(\left|F_{\varepsilon}(r, \mu, t)-F_{0}(r, \mu, t)\right|+\left|\sigma_{\varepsilon}(t)-\mathcal{J}_{0}(t)\right|\right)=0 ; \\
& \sup _{1 \geq \varepsilon>0,0 \leq t \leq T, \mu \in \mathcal{C}}\left|\sigma_{\varepsilon}^{-1}(t)\right|<\infty ; \\
& \lim _{\varepsilon \downarrow 0} \sup _{|r|>\delta} \sup _{0 \leq t \leq T, \mu \in \mathcal{C}}\left|\tilde{D}_{\varepsilon}(r, t)\right|=0 ; \\
& \limsup _{\eta \downarrow 0} \sup _{r \in \mathcal{K}} \sup _{\mu \in \mathcal{C}}\left|F_{0 \leq s, t \leq T,|t-s| \leq \eta}(r, \mu, t)-F_{0}(r, \mu, s)\right|+\left|\mathcal{J}_{0}(t)-\mathcal{J}_{0}(s)\right|=0 .
\end{aligned}
$$

(iii) Given the solution $X_{0, \infty}(t)$ of $(1.7)$, suppose that $D_{0, k \ell}(\cdot, \cdot)$ and $F_{0, k}\left(\cdot, X_{0, \infty}(\cdot), \cdot\right)$ as functions of $(r, t)$ are both twice continuously differentiable, where $k, \ell=1, \ldots, d$.

For $m \in \mathbf{N}$ set

$$
\Lambda_{m}:=\left\{\left(p^{1}, \ldots, p^{m}\right) \in \mathbf{R}^{d \cdot m}: \exists i \neq j, i, j \in\{1, \ldots, m\}, \quad \text { with } p^{i}=p^{j}\right\} .
$$

Infinite sequences in $\mathbf{R}^{d}$ will be denoted by either $\left(r^{1}, r^{2}, \ldots\right)$ or $r^{(\cdot)}$. The corresponding state space, $\left(\mathbf{R}^{d}\right)^{\infty}$, will be endowed with the metric

$$
d_{\infty}\left(r^{(\cdot)}, q^{(\cdot)}\right):=\sum_{k=1}^{\infty} 2^{-k} \rho\left(r^{k}-q^{k}\right)
$$

$C([0, \infty) ; \mathbf{M})$ denotes the space of $\mathbf{M}$-valued continuous functions, where $\mathbf{M}$ is some metric space.

\footnotetext{
${ }^{9}$ Under the additional assumption (5.7) in the following Section 5, the Stratonovich form of (1.19) does not produce a correction term. However, the chain rule becomes the simple chain rule and leads to the first-order SPDE (1.21).

${ }^{10}$ The third assumption in what follows requires that the correlation matrix $\tilde{D}_{\varepsilon}(q-p, t)_{\mid q-p=r}$ must tend to 0 , as the correlation length $\varepsilon$ tends to 0 . (Cf. (1.9) for a more general correlation matrix.) That is, in the limit the noise is spatially uncorrelated. The other assumptions are technical.
} 
Theorem 1.3. Let $\mathcal{X}_{\varepsilon}(\cdot)$ denote the solution of (1.21). Suppose (2.4) in addition to Hypothesis $1.2,(4.5),(5.5)$ and (5.7). Further, suppose that $d \geq 2$ and that $\left\{q_{\varepsilon}^{1}, q_{\varepsilon}^{2}, \ldots ..\right\}$ is a sequence of exchangeable initial conditions in (1.13) and (1.4), respectively, such that for all $m \in \mathbf{N}$ and $\varepsilon \geq 0$,

$$
P\left\{\left(q_{\varepsilon}^{1}, \ldots, q_{\varepsilon}^{m}\right) \in \Lambda_{m}\right\}=0,
$$

where $q_{\varepsilon}^{1}, \ldots, q_{\varepsilon}^{m}$ are the initial conditions in (1.13) for $\varepsilon>0$ and in (1.4) for $\varepsilon=0$, respectively. Finally, suppose

$$
\left(\mathcal{X}_{\varepsilon}(0), q_{\varepsilon}^{1}, q_{\varepsilon}^{2}, \ldots . .\right) \Rightarrow\left(X_{0, \infty}(0), q_{0}^{1}, q_{0}^{2}, \ldots . .\right) \quad \text { in } \mathbf{M}_{1} \times\left(\mathbf{R}^{d}\right)^{\infty}, \quad \text { as } \varepsilon \downarrow 0,
$$

where $\mathcal{X}_{\varepsilon}(0)$ are the initial values for $(1.21)$ and $X_{0, \infty}(0)=\lim _{N \rightarrow \infty} \frac{1}{N} \sum_{j=1}^{N} \delta_{q_{0}^{j}}$ is the initial condition of (1.7). Then

$$
\mathcal{X}_{\varepsilon}(\cdot) \Rightarrow X_{0, \infty}(\cdot) \text { in } C\left([0, \infty) ; \mathbf{M}_{1}\right) \text { as } \varepsilon \downarrow 0 .
$$

Proof. The main steps in the proof of the Kotelenez-Kurtz result are:

- Relative compactness criteria, established in Ethier and Kurtz (loc. cit.);

- In dimension $d \geq 2$, with probability 1 , the limiting Brownian motions do not intersect (cf. Friedman [8]);

- A generalization of de Finnetti's theorem due to T. Kurtz.

- The representation of the Itô SPDE (1.19) as the Stratonovich SPDE (1.21), derived in our Theorem 5.3 of Section 5 .

REMARK 1.4. (i) The importance of Theorem 1.3 comes from the well-known WongZakai approximation result (cf. Wong and Zakai [38]) SODE's, replacing the driving Brownian motion by piecewise linear approximations. We expect that this result can be generalized to the Stratonovich SPDE (1.21) uniformly in $\varepsilon$. Assuming that this is possible, we can obtain a semi-linear second-order parabolic PDE as the limit of first order random PDE's as the random drift (associated with $\mathcal{J}_{\varepsilon}$ ) tends to $\infty$.

(ii) Most of the results mentioned in this section have been obtained in much more generality by numerous authors. We just mention the following: The SPDE (1.13) has been solved Kotelenez [18] for finite signed measures (Kotelenez [18] and also for a class of $\sigma$-finite measures (Kotelenez 21]). Kurtz and Xiong 27] also employ the particle approach where the mass is not conserved.

(iii) The extension of the macroscopic limit theorem to finite positive measures, given mass conservation, is trivial. An extension of this result to signed measures is planned. Such an extension would establish the stochastic Navier Stokes equation for the vorticity distribution in 2D fluid mechanics, studied in Kotelenez [17, as a mesoscopic equation, converging to the solution of the corresponding macroscopic PDE.

(iv) Under certain assumptions the limit $\varepsilon \rightarrow 0$ for $d=1$ yields a measure diffusion process with clumping of the large particles. Cf. Dorogovtsev [3] and the references therein.

Sections 2 and 4 contain some additional facts and definitions with respect to SODE's and SPDE's. respectively. Section 3 provides qualitative results on correlated Brownian motions, which can be represented as solutions of a somewhat generalized form of (1.2) (cf. (3.1) and Proposition 3.1). In Section 5 we derive the Stratonovich form SODE's, 
driven by correlated Brownian motions and prove a chain rule representation, leading to the first-order SPDE's (1.21).

2. Stochastic ordinary differential equations. We define a metric on $\mathbf{R}^{d}, d \geq 1$, by

$$
\rho(r-q):=|r-q| \wedge 1,
$$

where $r, q \in \mathbf{R}^{d},|r-q|$ is the Euclidean distance on $\mathbf{R}^{d}$ and " $\wedge$ " denotes "minimum". We define a Wasserstein metric on $\mathbf{M}_{1}$ as follows:

The space of all continuous Lipschitz functions $f$ from $\mathbf{R}^{d}$ into $\mathbf{R}$ will be denoted by $C_{L}\left(\mathbf{R}^{d} ; \mathbf{R}\right)$. Further, $C_{L, \infty}\left(\mathbf{R}^{d} ; \mathbf{R}\right)$ is the space of all uniformly bounded Lipschitz functions $f$ from $\mathbf{R}^{d}$ into $\mathbf{R}$. Abbreviate

$$
\left|\left\|f \left|\| : = \operatorname { s u p } _ { q } | f ( q ) | ; \quad \| f \| _ { L } : = \operatorname { s u p } _ { \{ r \neq q , | r - q | \leq 1 \} } \frac { | f ( r ) - f ( q ) | } { \rho ( r - q ) } ; \quad \| f \| _ { L , \infty } : = \| f \left\|_{L}+|\|f \mid\| .\right.\right.\right.\right.
$$

For $\mu, \nu \in \mathbf{M}_{1}$, we set

$$
\gamma(\mu-\nu):=\sup _{\|f\|_{L, \infty} \leq 1}\left|\int f(q)(\mu(d q)-\nu(d q))\right| .
$$

Note that $\left(\mathbf{M}_{1}, \gamma\right)$ is a complete and separable metric space. (1.4) and (1.8) can be linked by defining an $\mathcal{M}_{d \times d^{-}}$valued function $\sigma^{\perp}(r, \mu, t)$ and a family of i.i.d. standard $\mathbf{R}^{d}$-valued Brownian motions, $\left\{\beta^{\perp, n}(\cdot)\right\}_{n \in \mathbf{N}}$, which is independent of $\left\{w_{\ell}\right\}_{\ell=1, . ., d} 11$ Endowing the domains and ranges of $F, \mathcal{J}$ and $\sigma^{\perp}$ with the Borel $\sigma$-algebras, we assume $F, \mathcal{J}$ and $\sigma^{\perp}$ to be jointly measurable in all variables. Then the motion of $N$ particles is described by the following system of SODE's:

$$
\begin{gathered}
\left.\begin{array}{c}
d r_{\varepsilon, N}^{i}=F_{\varepsilon}\left(r_{\varepsilon, N}^{i}(t), \mathcal{X}_{\varepsilon, N}(t), t\right) d t \\
\quad+\int \mathcal{J}_{\varepsilon}\left(r_{\varepsilon, N}^{i}(t), q, \mathcal{X}_{\varepsilon, N}(t), t\right) w(d q, d t)+\sigma^{\perp}\left(r_{\varepsilon, N}^{i}(t), \mathcal{X}_{\varepsilon, N}(t), t\right) d \beta^{\perp, i}, \\
r_{\varepsilon, N}^{i}(0)=q_{\varepsilon}^{i}, i=1, \ldots, N, \quad \mathcal{X}_{\varepsilon, N}(t):=\sum_{i=1}^{N} \frac{1}{N} \delta_{r_{\varepsilon, N}^{i}}(t) .
\end{array}\right\}
\end{gathered}
$$

Let $c_{F}, c_{\mathcal{J}}, c_{\sigma} \in(0, \infty)$ and assume globally Lipschitz and boundedness conditions 12

$$
\begin{aligned}
& \left|F\left(r_{1}, \mu_{1}, t\right)-F\left(r_{2}, \mu_{2}, t\right)\right| \leq c_{F}\left\{\rho\left(r_{1}-r_{2}\right)+\gamma\left(\mu_{1}-\mu_{2}\right)\right\}, \\
& \sum_{k, \ell=1}^{d}\left[\int\left(\mathcal{J}_{k \ell}\left(r_{1}, p, \mu_{1}, t\right)-\mathcal{J}_{k \ell}\left(r_{2}, p, \mu_{2}, t\right)\right)^{2} d p+\left|\sigma_{k \ell}^{\perp}\left(r_{1}, \mu_{1}, t\right)-\sigma_{k \ell}^{\perp}\left(r_{2}, \mu_{2}, t\right)\right|^{2}\right] \\
& \leq c_{\mathcal{J}, \sigma}^{2}\left\{\rho^{2}\left(r_{1}-r_{2}\right)+\gamma^{2}\left(\mu_{1}-\mu_{2}\right)\right\}, \\
& \left|F_{\varepsilon}(r, \mu, t)\right|^{2}+\sum_{k, \ell=1}^{d} \int \mathcal{J}_{\varepsilon, k \ell}^{2}(r, p, \mu, t) d p \leq c_{F, \mathcal{J}},
\end{aligned}
$$

where $\left(r_{\ell}, \mu_{\ell}, t\right) \in \mathbf{R}^{d} \times \mathbf{M}_{1} \times \mathbf{R}, \quad \ell=1,2$. For examples of kernels satisfying the above assumptions we refer to Kotelenez [18, [21. Along with (2.3) we also consider the following system of SODE's, where the empirical process is replaced by an adapted,

\footnotetext{
${ }^{11}$ We consider $\sigma^{\perp}$ and $\left\{\beta^{\perp, n}(\cdot)\right\}_{n \in \mathbf{N}}$ different from the corresponding quantities in (1.4) since the latter ones will be needed to describe the macroscopic limit for (1.11).

${ }^{12}$ For alternative assumptions including linear growth, cf. Kotelenez 21.
} 
continuous $\mathbf{M}_{1}$-valued process $\tilde{\mathcal{Y}}$ :

$$
\left.\begin{array}{rl}
d r_{\varepsilon, N}^{i}= & F_{\varepsilon}\left(r_{\varepsilon, N}^{i}(t), \tilde{\mathcal{Y}}(t), t\right) d t \\
& \quad+\int \mathcal{J}_{\varepsilon}\left(r_{\varepsilon, N}^{i}(t), q, \tilde{\mathcal{Y}}(t), t\right) w(d q, d t)+\sigma^{\perp}\left(r_{\varepsilon, N}^{i}(t), \tilde{\mathcal{Y}}(t), t\right) d \beta^{\perp, i}, \\
r_{\varepsilon, N}^{i}(0)= & q_{\varepsilon}^{i}, i=1, \ldots, N .
\end{array}\right\}
$$

Obviously, the equations for the particle positions in (2.5) are coupled only through the noise term $w(d r, d t)$ and do not depend on $N$. We can, of course, start the equations at some point $s \geq 0$, and we denote such a solution of (2.5), if it exists, by $r_{N}\left(t, \mathcal{Y}, r_{N, s}, s\right)$ and its $k$-th component by $r_{\varepsilon}^{k}$.

Let $\mathcal{G}_{s, t}\left(\right.$ resp. $\left.\mathcal{G}_{t}\right)$ be the $\sigma$-algebra generated by $w(d p, d u$ ) between $s$ and $t$ (resp. 0 and $t$ ) for $t \geq s$. By analogy, for $t \geq s, \mathcal{G}_{s, t}^{\perp}$ and $\mathcal{G}_{t}^{\perp}$ are the $\sigma$-algebras generated by $\left\{d \beta^{\perp, n}(u)\right\}_{n \in \mathbf{N}}$ between $s$ and $t$ and 0 and $t$, respectively. The cylinder set filtrations on $C\left([0, \infty) ; \mathbf{M}_{1}\right)$ are denoted $\mathcal{F}_{\mathbf{M}, s, t}$, respectively $\mathcal{F}_{\mathbf{M}, t}$, if $s=0$. Completed $\sigma$-algebras will be denoted with a bar on top of the $\sigma$-algebra, e.g. $\overline{\mathcal{G}}_{s, t}$. Further, if $f$ is a stochastic process on $[s, \infty)$ with values in some metric space, we set for $t \geq s$,

$$
\left(\pi_{s, t} f\right)(u):=f(u \wedge t),(u \geq s) .
$$

Theorem 2.1. Assume (2.4). Then:

1) (2.5) has a unique solution $r_{\varepsilon}^{k}\left(\cdot, \tilde{\mathcal{Y}}, r_{0}^{k}, 0\right) \in C\left([s, \infty) ; \mathbf{R}^{d}\right)$ a.s.

2) Let $\tilde{\mathcal{Y}}_{i}$ be continuous adapted measure processes and $r_{s, i}^{k}$ be adapted initial conditions, $i=1,2$. Then for any $T \geq s$,

$$
\left.\begin{array}{l}
E \sup _{s \leq t \leq T \wedge \tau} \rho^{2}\left(r_{\varepsilon}^{k}\left(t, \tilde{\mathcal{Y}}_{1}, r_{s, 1}^{k}, s\right), r^{k}\left(t, \tilde{\mathcal{Y}}_{2}, r_{s, 2}^{k}, s\right)\right) \\
\leq \quad c_{T, F, \mathcal{J}, \sigma}\left\{E\left(\rho^{2}\left(r_{s, 1}^{k}, r_{s, 2}^{k}\right)\right)+E \int_{s}^{T \wedge \tau}\left(\gamma^{2}\left(\tilde{\mathcal{Y}}_{1}(u)-\tilde{\mathcal{Y}}_{2}(u)\right) d u\right\} .\right.
\end{array}\right\}
$$

Further, with probability 1 uniformly in $t \in[s, \infty)$,

$$
r_{\varepsilon}^{k}\left(t, \tilde{\mathcal{Y}}, r_{s, 1}^{k}, s\right) \equiv r^{k}\left(t, \pi_{s, t} \tilde{\mathcal{Y}}, r_{s, 1}^{k}, s\right)
$$

3) For any $N \in \mathbf{N}$ there is an $\mathbf{R}^{d N}$-valued map in the variables $\left(t, \omega, \mu(\cdot), r_{N}, s\right)$, $0 \leq s \leq t<\infty$ such that for any fixed $s \geq 0$,

$$
\left.\bar{r}_{N}(\cdot, \ldots, \cdot, s): \Omega \times C([s, T]) ; \mathbf{M}_{1}\right) \times \mathbf{R}^{d N} \rightarrow C\left([s, T] ; \mathbf{R}^{d N}\right),
$$

and the following holds:

(i) For any $t \in[s, T], \bar{r}_{N}(t, \cdot, \ldots, \cdot, s)$ is $\overline{\mathcal{G}}_{s, t} \otimes \overline{\mathcal{G}}_{s, t}^{\perp,[1, N]} \otimes \mathcal{F}_{\mathbf{M}, s, t} \otimes \mathcal{B}^{d N} \otimes \mathcal{B}_{[s, t]}-\mathcal{B}^{d N}-$ measurable.

(ii) The $i$-th $d$-vector of $\bar{r}_{N}=\left(\bar{r}^{1}, \ldots, \bar{r}^{i}, \ldots \bar{r}^{N}\right)$ depends only on the $i$-th $d$-vector initial condition $r_{s}^{i} \in \mathcal{R}_{2, s}^{d}$ and the Brownian motion $\beta^{\perp, i}(\cdot)$ in addition to its dependence on $w(d q, d t)$ and $\tilde{\mathcal{Y}}$, and with probability 1 (uniformly in $t \in[s, \infty)$ )

$$
\bar{r}_{N}^{i}\left(t, \cdot, \tilde{\mathcal{Y}}, r_{s}^{i}, s\right) \equiv r_{\varepsilon}^{i}\left(t, \tilde{\mathcal{Y}}, r_{s}^{i}, s\right) .
$$

(iii) If $u \geq s$ is fixed, then with probability 1 (uniformly in $t \in[u, \infty)$ ),

$$
\left.\begin{array}{l}
\bar{r}_{N}\left(t, \cdot, \pi_{u, t} \tilde{\mathcal{Y}}, \bar{r}_{N}\left(u, \cdot, \pi_{s, u} \tilde{\mathcal{Y}}, r_{N, s}, s\right), u\right) \\
\equiv \bar{r}_{N}\left(t, \cdot, \pi_{s, t} \mathcal{\mathcal { Y }}, r_{N, s}, s\right) .
\end{array}\right\}
$$


Proof. By our global Lipschitz assumptions we obtain existence, uniqueness and (2.6) from the usual iteration, employing Gronwall's inequality and the contraction mapping principle. (2.7) follows immediately from the construction. Statement 3 is proved in Section 6 of Kotelenez [21], following the construction of Markov solutions which are jointly measurable in $(\omega, q, t)$ for SODE's, as given in Dynkin [5, Ch. 11, Section 2.

Next, we consider the $\mathbf{R}^{d N}$-valued system of coupled SODE's (2.3). We endow $\mathbf{R}^{d N}$ with the metric

$$
\rho_{N}\left(r_{N}, q_{N}\right):=\max _{1 \leq i \leq N} \rho\left(r_{i}, q_{i}\right),
$$

where $r_{N}:=\left(r^{1}, \ldots, r^{N}\right), q_{N}:=\left(q_{1}, \ldots, q_{N}\right) \in \mathbf{R}^{d N}$.

Theorem 2.2. Assume (2.4). Then, to each adapted initial condition $r_{N}(0) \in \mathbf{R}^{d N}$, (2.3) has a unique solution $r_{N}\left(\cdot, r_{N}(s)\right) \in C\left([s, \infty) ; \mathbf{R}^{N d}\right)$ a.s. which is a Markov process on $\mathbf{R}^{d N}$.

Proof. The proof is a simple alteration of the proof of Theorem 2.1.

3. Correlated Brownian motions. Recall the $N$-particle motion described by (1.2). Assuming Lipschitz conditions on $G_{\varepsilon}$ as in (2.4), Theorem 2.1 or (2.2) imply that (1.2) has a unique solution for every adapted and square integrable initial condition $r^{i}(0), i=1, \ldots, N$. Let us now generalize (1.2) to the following system of SODE's in integral form which is a special case of equations (2.5) and (2.3):

$$
r\left(t, r_{0}^{i}\right)=r_{0}^{i}+\int_{0}^{t} \int \Gamma_{\varepsilon}\left(r\left(s, r_{0}^{i}\right), q, t\right) w(d q, d t), \quad i=1, \ldots, N
$$

As in (2.3) and (2.5), $w(d q, d t)$ is a $d$-dimensional space-time white noise and $\Gamma_{\varepsilon}(r, q)$ is an $\mathcal{M}_{d \times d^{-v}}$ valued function such that (2.4) holds with $\Gamma_{\varepsilon}$ replacing $\mathcal{J}_{\varepsilon}$. Denote the square integrable continuous martingales from the right-hand side of (3.1) by

$$
M_{\varepsilon}\left(t, r_{0}^{i}\right):=\int_{0}^{t} \int \Gamma_{\varepsilon}\left(r\left(u, r_{0}^{i}\right), q\right) w(d q, d u), \quad i=1, \ldots, N .
$$

We may use the increments of $M_{\varepsilon}\left(t, r_{0}^{i}\right)$ as a stochastic perturbation of a deterministic ODE. Of particular interest are the special cases of (2.3) or (2.5) if we replace $\mathcal{J}$ by $\Gamma_{\varepsilon}$ and assume $\sigma^{\perp} \equiv 0$. We may simplify the set of SODE's even further, assuming the initial conditions to be deterministic points $r_{0}^{i}$ at time $t=0$ and $r_{0}^{i} \neq r_{0}^{j}$ if $i \neq j$. We next describe the correlations of $M_{\varepsilon}\left(\cdot, r_{0}\right)$ from $(3.2)$, where the second variable is the initial condition from the continuum $\mathbf{R}^{d}$, which is assumed to be deterministic. To this end, we recall the definition of the tensor quadratic variation of a vector-valued martingale, as given by Metivier and Pellaumail 29, Section 2.3. In the case of finite-dimensional martingales, the tensor quadratic variation reduces to the mutual quadratic variations of all components of the vector-valued martingale. The tensor quadratic variation of the 
$\mathbf{R}^{2 d}$-valued continuous square integrable martingale $\left(M\left(t, r_{0}\right), M\left(t, \tilde{r}_{0}\right)\right)$ is given by

$$
\begin{array}{r}
{\left[M_{k}\left(t, r_{0}\right), M_{\ell}\left(t, \tilde{r}_{0}\right)\right]=\int_{0}^{t} \int \sum_{m=1}^{d} \Gamma_{\varepsilon, k m}\left(r\left(u, r_{0}\right), q\right) \Gamma_{\varepsilon, \ell m}\left(r\left(u, \tilde{r}_{0}\right), q\right) d q d u,} \\
k, \ell=1, \ldots, d, r_{0}, \tilde{r}_{0} \in \mathbf{R}^{d} .
\end{array}
$$

(3.3) has a derivative in $t$. It follows from Itô's formula that this derivative serves as the diffusion matrix in an equation for the mass distribution associated with (3.1):

$$
D_{\varepsilon, k \ell}(r, \tilde{r}):=\int \sum_{m=1}^{d} \Gamma_{\varepsilon, k m}(r, q) \Gamma_{\varepsilon, \ell m}(\tilde{r}, q) d q, \text { where } r\left(t, r_{0}\right)=r, \quad r\left(t, \tilde{r}_{0}\right)=\tilde{r} .
$$

The diffusion matrix in $\mathcal{M}_{(2 d) \times(2 d)}$, the space of $(2 d \times 2 d)$-matrices, consists of 4 $(d \times d)$-matrices which describe the spatial pair correlations between the motions of two given particles, indexed by $r_{0}$ and $\tilde{r}_{0}$, at a given time. Of special interest are the block diagonal $(d \times d)$-matrices, i.e., when $r_{0}=r_{0}$ or $\tilde{r}_{0}=\tilde{r}_{0}$, in particular, for the case of spatially homogeneous diffusion coefficients, i.e., when the diffusion coefficients satisfy

$$
D_{\varepsilon, k \ell}(r, q)=D_{\varepsilon, k \ell}(r-q) \quad \forall r, q .
$$

If $r_{0}=\tilde{r}_{0}$ and the diffusion matrix is homogeneous, the uniqueness of the solutions implies that the tensor quadratic variation for one given particle is a constant matrix times $t$. By (3.4) a sufficient (but not necessary) condition for the diffusion matrix to be homogeneous is that the kernel function $\Gamma_{\varepsilon}$ be spatially homogeneous, i.e.,

$$
\Gamma_{\varepsilon}(r, q)=\Gamma_{\varepsilon}(r-q) \quad \forall r, q .
$$

Proposition 3.1. Suppose the diffusion matrix associated with (3.1) is spatially homogeneous. Then, for each $r_{0}, M\left(\cdot, r_{0}\right)$, defined by $(3.2)$, is a $d$-dimensional Brownian motion with diffusion matrix with entries

$$
D_{\varepsilon, k \ell}(0), \quad k, \ell=1, \ldots, d .
$$

Proof. The proposition is a direct consequence of a $d$-dimensional generalization of Paul Levy's theorem (cf. Ethier and Kurtz [7, Chapter 7, Theorem 1.1).

Henceforth we will assume that the diffusion matrix, associated with (3.1), is spatially homogeneous, i.e., that (3.5) holds. To derive the space-time correlations for $\frac{d}{d t} M\left(\cdot, r_{0}\right)$, treated as a generalized space-time random field, we generalize the classical analysis of Brownian motion and its generalized derivative by Gel'fand and Vilenkin [10. It follows that $\frac{d}{d t} M(\cdot, \cdot, \omega) \in \mathcal{S}^{\prime}\left(\mathbf{R}^{d+1}\right)$, which is the dual of the infinitely often, rapidly decreasing real-valued functions on $\mathbf{R}^{d+1}$, denoted $\mathcal{S}\left(\mathbf{R}^{d+1}\right)$ (cf. Kotelenez 21], Section 15). Choosing $\varphi_{d}, \psi_{d} \in \mathcal{S}\left(\mathbf{R}^{d}\right)$ and $\varphi, \psi \in \mathcal{S}(\mathbf{R})$, we obtain the following random covariance for the space-time random field $\frac{d}{d t} M(\cdot, \cdot)$ :

$$
\begin{aligned}
& \operatorname{Cov}_{d+1, \omega}\left[\left(\varphi_{d} \varphi, \frac{d}{d t} M_{\varepsilon}(\cdot, \cdot)\right)\left(\psi_{d} \psi, \frac{d}{d t} M_{\varepsilon}(\cdot, \cdot)\right)\right] \\
& =\iiint_{0}^{\infty} \int_{0}^{\infty} \varphi_{d}\left(r_{0}\right) \psi_{d}\left(\tilde{r}_{0}\right) \varphi(t) \psi(s) \delta_{0}(t-s) \int \sum_{m=1}^{d} \Gamma_{\varepsilon, k m}\left(r\left(t, r_{0}, \omega\right), q\right) \\
& \quad \times \Gamma_{\varepsilon, \ell m}\left(r\left(s, \tilde{r}_{0}, \omega\right), q\right) d q d s d t d r_{0} d \tilde{r}_{0} .
\end{aligned}
$$


Hence, the random covariance is an $\mathcal{M}_{d \times d^{-v a l u e d}}$ bilinear functional on $\mathcal{S}\left(\mathbf{R}^{d+1}\right)$ :

$$
\operatorname{Cov}_{d+1, \omega}=\delta_{t-s} \otimes D_{\varepsilon}\left(r\left(t, r_{0}, \omega\right)-r\left(s, \tilde{r}_{0}, \omega\right)\right) .
$$

The space-time correlations for $M(\cdot, \cdot), \overline{\operatorname{Cov}}_{d+1}$, are obtained by taking the mathematical expectation in (3.8):

$$
\overline{\operatorname{Cov}}_{d+1}=\delta_{t-s} \otimes E\left(D_{\varepsilon}\left(r\left(t, r_{0}\right)-r\left(s, \tilde{r}_{0}\right)\right)\right) .
$$

Observe that the joint motion of $\left(M\left(\cdot, r_{0}\right), M\left(\cdot, \tilde{r}_{0}\right)\right)$ for $r_{0} \neq \tilde{r}_{0}$ is not Gaussian. We are now ready to formulate the main concept of this section:

Definition 3.2. Suppose the diffusion matrix associated with (3.1) is spatially homogeneous. The system of $N d$-dimensional martingales $M\left(\cdot, r^{i}\right)$, defined by $(3.2)$, $i=1, \ldots, N$ are be called " $N d$-dimensional correlated Brownian motions starting at 0 " and the pair space-time correlation is given by (3.9). If, in addition, the initial conditions in (3.1) are deterministic, the system of solutions $r\left(\cdot, r_{0}^{i}\right), i=1, \ldots, N$, are called " $N$ d-dimensional correlated Brownian motions starting at $\left(r_{0}^{1}, \ldots, r_{0}^{N}\right)$ ".

In the following proposition we allow adapted random initial conditions. We then show that particles, performing correlated Brownian motions and starting with positive probability at the same point, will stick together with the same probability.

Proposition 3.3. Let $A:=\left\{\omega: r^{i}(0, \omega)=r^{j}(0, \omega)\right\}$ and assume $1>P(A)>0$. Then $\forall t \geq 0$,

$$
r\left(t, \omega, r_{0}^{i}\right)=r\left(t, \omega, r_{0}^{j}\right) \quad \text { if } \omega \in A, \text { and } r\left(t, \omega, r_{0}^{i}\right) \neq r\left(t, \omega, r_{0}^{j}\right) \forall t \text { if } \omega \notin A \text {. }
$$

Proof. The solutions of (3.1) are unique, which implies that starting at the same point, the positions of the particles will not separate. That particles with starts in different positions never collide follows from a similar statement and a proof provided by N. Krylov 24].

We need the following result about shift and rotational invariance. Denote by $\mathcal{O}(d)$ the orthogonal $(d \times d)$-matrices over $\mathbf{R}^{d}$, i.e., $\mathcal{O}(d):=\left\{Q \in \mathcal{M}_{d \times d}: \operatorname{det}(\mathrm{Q})= \pm 1\right\}$. Let $h \in \mathbf{R}^{d}$ and $Q \in \mathcal{O}(d)$. Let $A$ be a bounded Borel set in $\mathbf{R}^{d}$ and define the spatially shifted (resp. rotated) space-time white noise by:

$$
\left.\begin{array}{l}
\int_{A} w_{-h}(d q, d s):=\int_{A+h} w(d q, d s), \\
\int_{A} w_{Q^{-1}}(d q, d s):=\int_{Q A} w(d q, d s) .
\end{array}\right\}
$$

Kotelenez [21, Section 5.2, proves that the following (generalized) random fields are equivalent in distribution. Denoting this equivalence by " $\sim$ ", we have

$$
\int_{0}^{\cdot} w(d q, d s) \sim \int_{0}^{\cdot} w_{Q^{-1}}(d q, d s) \sim \int_{0}^{\cdot} w_{-h}(d q, d s) .
$$

We will now restrict our analysis to two correlated Brownian motions, $r\left(\cdot, r_{0}^{i}\right), i=1,2$, as described by (1.2). Setting $G_{\varepsilon}=\Gamma_{\varepsilon, \cdot 1}$ and assuming that the other column vectors of $\Gamma_{\varepsilon}$ are identically 0 in addition to spatial homogeneity of $G_{\varepsilon},(3.1)$ reduces to (1.2), where we now use the notation $w(d q, d t)$ to denote a scalar-valued space-time white noise.

We denote the solutions of (1.2) with initial conditions $r_{0}^{i}, i=1,2$, and driving noise $w(d r, d t)$ by $r^{1}(\cdot):=r\left(\cdot, r_{0}^{1}, w\right)$ and $\left.r^{2}(\cdot):=r\left(\cdot, r_{0}^{2}, w\right)\right)$, respectively. By the shift 
invariance of the kernel $G_{\varepsilon}$, (3.12) implies the shift invariance of the solutions if the initial conditions are shifted by the same vector $h \in \mathbf{R}^{d}$. To obtain a similar statement for rotations we follow Truesdell and Knoll 35, Sec. 17, and call functions $\vartheta: \mathbf{R}^{d} \longrightarrow \mathbf{R}$, $G: \mathbf{R}^{d} \longrightarrow \mathbf{R}^{d}$ and $A: \mathbf{R}^{d} \longrightarrow \mathcal{M}_{d \times d}$ "frame-indifferent" if

$$
\vartheta(Q r)=\vartheta(r), \quad G(Q r)=Q G(r), \quad A(Q r)=Q A(r) Q^{T} \quad \forall r \in \mathbf{R}^{d}, Q \in \mathcal{O}(d) .
$$

To describe the structure of matrix-valued frame-indifferent functions, we first denote the subspace spanned by $r,\{r\}$, and the subspace orthogonal to $\{r\}$, we denote $\{r\}^{\perp}$. Let $I_{d}$ be the identity matrix in $\mathbf{R}^{d}$. Then we define the projection operators

$$
P(r):=\frac{r r^{T}}{|r|^{2}}, \quad P^{\perp}(r):=I_{d}-P(r),
$$

where $r r^{T}$ is the matrix product between the column vector $r$ and the row vector $r^{T}$, resulting in the $(d \times d)$-matrix with entries $r_{k} r_{\ell}, k, \ell=1, \ldots, d$. Assuming $A$ is symmetric, a proof by M. Leitman (cf. Kotelenez, Leitman and Mann 22]) shows that these functions are frame-indifferent if and only if there are scalar functions $\alpha, \beta, \lambda, \lambda^{\perp}: \mathbf{R}_{+} \longrightarrow \mathbf{R}$ such that

$$
\begin{array}{r}
\vartheta(r) \equiv \alpha\left(|r|^{2}\right), \quad G(r) \equiv \beta\left(|r|^{2}\right) r, \quad A(r) \equiv \lambda\left(|r|^{2}\right) P(r)+\lambda^{\perp}\left(|r|^{2}\right) P^{\perp}(r) \\
\forall r \in \mathbf{R}^{d}, Q \in \mathcal{O}(d) .
\end{array}
$$

We easily verify that the kernel in (1.3), associated with a Maxwellian velocity field, is shift-invariant and, by (3.15), also frame-indifferent.

We quote from Kotelenez, Leitman and Mann 22] (cf. also Kotelenez [21]).

Proposition 3.4.

$$
\left.\begin{array}{c}
\left(r\left(\cdot, r_{0}^{1}, w\right)+h, r\left(\cdot, r_{0}^{2}, w\right)+h\right) \sim\left(r\left(\cdot, r_{0}^{1}+h, w\right), r\left(\cdot, r_{0}^{2}+h, w\right)\right), \\
\text { and, if } G_{\varepsilon} \text { is frame-indifferent, also } \\
\left(Q r\left(\cdot, r_{0}^{1}, w\right), Q r\left(\cdot, r_{0}^{2}, w\right)\right) \sim\left(r\left(\cdot, Q r_{0}^{1}, w\right), r\left(\cdot, Q r_{0}^{2}, w\right)\right),
\end{array}\right\}
$$

where the pair processes are considered as $C\left([0, \infty) ; \mathbf{R}^{2 d}\right)$-valued random variables.

Note that (3.16) is only correct if we shift both $r^{1}$ and $r^{2}$ by the same $d$-dimensional vector $h$, or rotate them by the same orthogonal matrix $Q$.

We are interested in effects of the diffusion coefficient on the motion $\left|r^{2}(t)-r^{1}(t)\right|$, which will be called the "magnitude of the separation". An initial step in this direction is a change of coordinates. First we will employ the shift invariance to obtain a Markov representation for $r\left(\cdot, r_{0}^{2}\right)-r\left(\cdot, r_{0}^{1}\right)$. Set

$$
\left.\begin{array}{c}
\bar{D}_{\varepsilon, k \ell}(\sqrt{2} q):=\left[\tilde{D}_{\varepsilon, k \ell}(0)-\tilde{D}_{\varepsilon, k \ell}(\sqrt{2} q)\right], \\
\tilde{D}_{\varepsilon, k \ell}(\sqrt{2} q):=\int G_{\varepsilon, k}(q-\tilde{q}) G_{\varepsilon, \ell}(-\tilde{q}) d \tilde{q}, \quad k, \ell=1, . ., d .
\end{array}\right\}
$$

Kotelenez, Leitman and Mann (loc. cit.) show that the frame-indifference implies the existence of scalar functions

$$
\alpha_{\varepsilon}, \alpha_{\perp, \varepsilon}: \mathbf{R}_{+} \rightarrow \mathbf{R} ; \xi \mapsto \alpha_{\varepsilon}(\xi), \alpha_{\perp, \varepsilon}
$$

such that there is a constant $c_{\varepsilon}>0$ and the following holds:

$$
\alpha_{\varepsilon}(0)=0=\alpha_{\perp, \varepsilon}(0) \quad \text { and } \quad \lim _{|r| \rightarrow \infty} \alpha_{\varepsilon}\left(|r|^{2}\right)=c_{\varepsilon}=\lim _{|r| \rightarrow \infty} \alpha_{\perp, \varepsilon}\left(|r|^{2}\right)
$$


and

$$
\bar{D}_{\varepsilon}(\sqrt{2} r)=\alpha_{\perp, \varepsilon}\left(|r|^{2}\right) P^{\perp}(r)+\alpha_{\varepsilon}\left(|r|^{2}\right) P(r) .
$$

Employing this representation and Proposition 3.3, Kotelenez, Leitman and Mann (loc. cit.) prove the following.

Theorem 3.5. Set $x_{0}:\left|r_{0}^{2}-r_{0}^{1}\right|$ and suppose $d>1$. Then the magnitude of the separation process is equivalent in distribution to the solution of the following stochastic ordinary differential equation:

$$
d x=\frac{1}{2}(d-1) \frac{\alpha_{\perp, \varepsilon}\left(x^{2}\right)}{x} d t+\sqrt{\alpha_{\varepsilon}\left(x^{2}\right)} \beta(d t), \quad x(0)=x_{0},
$$

where $\beta(\cdot)$ is a one-dimensional standard Brownian motion.

We first want to describe the long-time behavior of the magnitude of the separation and then briefly comment on its short-time behavior. For $x>0$ define functions

$$
\psi(x) \in\{\log (x+e), 1(x)\},
$$

where $1(x) \equiv 1, \log (\cdot)$ is the natural $\log$ arithm and $\log (e)=1$. Suppose

$$
\int\left|G_{k \ell}(-p)\right|^{2} \psi^{2}(|p|) d p<\infty \quad \forall k, \ell .
$$

Recall in the following theorem that for $d \geq 2, x\left(t, x_{0}\right) \sim\left|r\left(t, r^{2}\right)-r\left(t, r^{1}\right)\right|_{\left\{x_{0}=\left|r^{2}-r^{1}\right|\right\}}$, where $r\left(\cdot, r^{i}\right), i=1,2$, are solutions of $(2.9)$. For $d=1$, we have $r(t, q) \sim$ $\left(r\left(t, r^{2}\right)-r\left(t, r^{1}\right)\right)_{\left\{q=r^{2}-r^{1}\right\}}$

Theorem 3.6. 13 Suppose (3.22) with $\psi(x) \equiv 1$ if $d \neq 2$ and $\psi(x)=\log (x+e)$ if $d=2$. Further, suppose that the diffusion matrix with entries $\bar{D}_{\varepsilon}(\sqrt{2} r)$ is positive definite for $r \neq 0$. Finally, assume for $d=2$ that $\alpha_{\varepsilon}(\xi)>0 \forall \xi>0$. Then the following holds:

(i) $\{0\}$ is a.s. an attractor for the $r(\cdot)$ if $d=1$.

(ii) For $d=2$ and $x_{0} \neq 0$ the solution of (3.20) is recurrent.

(iii) For $d \geq 3$ and $x_{0} \neq 0$ the solution of (3.20) is transient.

As a consequence, if $d=1$, the two Brownian particles $r\left(\cdot, r^{i}\right), i=1,2$, will eventually clump. Further, if $d=2$, the two Brownian particles $r\left(\cdot, r^{i}\right), i=1,2$, will attract and repel each other infinitely often and, if $d \geq 3$, the distance between the particles will tend to $\infty$ with probability 1 , as $t \longrightarrow \infty$.

Proof. Case $d=1$ was proved by Kotelenez [20].

Case $d \geq 2$.

Assuming $\alpha_{\varepsilon}(\xi)>0 \forall \xi \geq \rho \geq 0$, the following functional (3.23) was used by Gikhman and Skorokhod [1], Ch. 4, Section 16, to study the asymptotic behavior of solutions of

\footnotetext{
${ }^{13}$ The cases $d \geq 2$ essentially state that for large $t$, correlated Brownian motions behave like uncorrelated (or independent) Brownian motions. This is possible since for $d \geq 2$ the limiting uncorrelated Brownian motions do not intersect. See the sketch of the proof of Theorem 1.3. The long-time behavior for $d=1$ is different from the long-time behavior of uncorrelated Brownian motions, as correlated Brownian motions for $d=1$ clump. This observation is consistent with the behavior of the corresponding SPDE's studied by Dorogovtsev [3]. See our preceding Remark 1.4, (iv).
} 
one-dimensional stochastic ordinary differential equations. For the notation, cf. Ikeda and Watanabe (loc. cit.), Ch. VI.3:

$$
s(x, \rho):=\int_{\rho}^{x} \exp \left[-\int_{\rho}^{y} \frac{(d-1) \alpha_{\perp, \varepsilon}\left(z^{2}\right)}{z \alpha_{\varepsilon}\left(z^{2}\right)} d z\right] d y .
$$

It follows that there are $-\infty<s^{-} \leq s^{+}<\infty$ such that

$$
\left.\begin{array}{cc}
s(x, \xi) \longrightarrow \pm \infty, \text { as } x \longrightarrow \pm \infty & \text { if } d=2, \\
s(x, \xi) \longrightarrow s^{ \pm}, \text {as } x \longrightarrow \pm \infty & \text { if } d>2 .
\end{array}\right\}
$$

Evoking Theorem 3.1 in Ikeda and Watanabe (loc. cit.) completes the proof.

REMARK 3.7. Generalizing the notion of probability flux in one dimension (cf. van Kampen [16]) to the divergence of the diffusion matrix $\bar{D}_{\varepsilon}(\sqrt{2} r)$ from (3.19), Kotelenez, Leitman and Mann (loc. cit.) show that for $d \geq 2$ that two correlated Brownian motions at close distance show attractive behavior for short times, which is in agreement with the depletion phenomenon.

4. Stochastic partial differential equations. For notational convenience we will drop the subscript " $\varepsilon$ " in this section. Recalling the definition of $D_{k \ell}(r, \mu, t)$ from $(1.12)$, we set

$$
\overline{\bar{D}}(r, \mu, t)=D(r, \mu, t)+\left(\sigma^{\perp}(r, \mu, t)\right)^{2} .
$$

THEOREM 4.1. In addition to (2.4), suppose that

$$
\mathcal{X}_{N}(0):=\frac{1}{N} \sum_{i=1}^{N} \delta_{r_{0}^{i}} \rightarrow \mathcal{X}_{0}, \text { in } E \gamma^{2}(\cdot-\cdot), \text { as } N \rightarrow \infty
$$

Then there is a unique continuous adapted $\mathbf{M}_{1}$-valued process $\mathcal{X}(\cdot)$ such that

$$
E \sup _{0 \leq t \leq T} \gamma\left(\mathcal{X}(t)-\mathcal{X}_{N}(t)\right) \rightarrow 0 \quad \forall T>0
$$

where $\mathcal{X}_{N}(\cdot)$ is the empirical process associated with $(2.3)$. Further, $\mathcal{X}(\cdot)$ is the solution of the following SPDE:

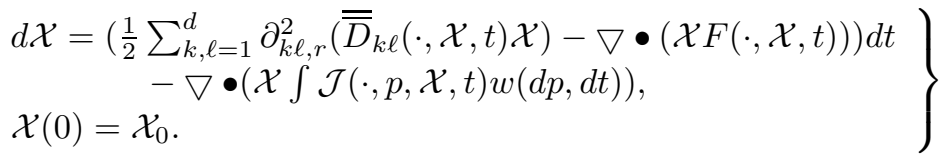

Sketch of Proof. (i) Let $\varphi \in C_{c}^{2}\left(\mathbf{R}^{d} ; \mathbf{R}\right)$, the real-valued twice continuously differentiable functions with compact support in $\mathbf{R}^{d}$. Integrating by parts in the generalized sense, we obtain by Itô's formula the generalization of (1.11):

$$
\begin{aligned}
& \left\langle d \mathcal{X}_{N}(t), \varphi\right\rangle=\left\langle\frac{1}{2} \sum_{k, \ell=1}^{d} \partial_{k \ell}^{2}\left(D_{k \ell}\left(\cdot, \mathcal{X}_{N}, t\right) \mathcal{X}_{N}(t)\right) d t, \varphi\right\rangle \\
& +\left\langle\frac{1}{2} \sum_{k, \ell=1}^{d} \partial_{k \ell}^{2}\left(\left(\sigma^{\perp}\right)_{k \ell}^{2}\left(\cdot, \mathcal{X}_{N}, t\right) \mathcal{X}_{N}(t)\right) d t, \varphi\right\rangle \\
& -\left\langle\nabla \bullet\left(\mathcal{X}_{N}(t) F\left(\cdot, \mathcal{X}_{N}, t\right)\right) d t, \varphi\right\rangle \\
& -\left\langle\nabla \bullet\left(\mathcal{X}_{N}(t) \int \mathcal{J}\left(\cdot, p, \mathcal{X}_{N}, t\right) w(d p, d t)\right), \varphi\right\rangle \\
& +\sum_{j=1}^{N} \frac{1}{N}(\nabla \varphi)\left(r^{j}(t)\right) \bullet\left(\sigma^{\perp}\left(r^{j}(t), \mathcal{X}_{N}, t\right) \beta^{\perp, j}(d t)\right)
\end{aligned}
$$


Exactly as in (1.6), Doob's inequality yields for the last term in (4.3):

$$
E \sup _{0 \leq t \leq T}\left(\int_{0}^{t} \sum_{j=1}^{N} \frac{1}{N}(\nabla \varphi)\left(r^{j}(s)\right) \bullet\left(\sigma^{\perp}\left(r^{j}(s), \mathcal{X}_{N}, s\right) \beta^{\perp, j}(d s)\right)\right)^{2} \leq O_{T, \sigma^{\perp}, \varphi}\left(\frac{1}{N}\right) .
$$

Therefore, if there is a limit $\mathcal{X}(\cdot)$ in the Wasserstein metric, uniformly on compact intervals, it must be a solution of (4.2).

(ii) To establish the existence of the continuum limit we analyze the behavior of the pair processes $\left(\left\{r^{1}(\cdot), \ldots, r^{N}(\cdot)\right\}, \mathcal{X}_{N}(\cdot)\right)$. We embed finite $\mathbf{R}^{d}$-valued sequences into infinite $\mathbf{R}^{d}$-valued sequences, using the one-point compactification $\hat{\mathbf{R}}^{d}$ of $\mathbf{R}^{d}$. Then, we extend the metric $\varrho$ to $\hat{\mathbf{R}}^{d}$, defining the distance between the added element $\diamond$ and $r$ to be 1. Hence, we can define a suitable product metric on $\left(\hat{\mathbf{R}}^{d}\right)^{\mathbf{N}} \times \mathbf{M}_{1}$. Based on our Lipschitz assumption (2.4) we can show that the pair processes $\left(\left\{r^{1}(\cdot), \ldots, r^{N}(\cdot), \diamond, \ldots\right\}, \mathcal{X}_{N}(\cdot)\right) \in$ $C\left([0, T] ;\left(\hat{\mathbf{R}}^{d}\right)^{\mathbf{N}} \times \mathbf{M}_{1}\right)$ are flows whose dependence on the initial conditions is uniformly continuous. Extension by continuity and projection onto the $\mathbf{M}_{1}$-valued processes yields the existence of a unique limit $\mathcal{X}(\cdot)$ (cf. Kotelenez [21]).

Alternatively, we can follow the procedure of Kurtz and Xiong (loc. cit.) which under exchangeability assumptions of the initial conditions of (2.3) evokes de Finnetti's theorem to establish the limit of $\mathcal{X}_{N}(\cdot)$.

To obtain smooth solutions of (4.2), the calculations are simpler if we assume that the diffusion matrix from $(1.9) /(1.12)$ is spatially homogeneous 14 i.e.,

$$
\left.\begin{array}{l}
\tilde{D}(r, q, \mu, t)=\tilde{D}(r-q, \mu, t), \\
D(r, \mu, t) \equiv \tilde{D}(0, \mu, t) .
\end{array}\right\}
$$

Spaces of interest for densities associated with smooth solutions of (4.2) include $L_{p}\left(\mathbf{R}^{d} ; d r\right)$ for $p=1,2$, the $p$-integrable real-valued functions with $d r$ the Lebesgue measure, where $H_{0}:=L_{2}\left(\mathbf{R}^{d} ; d r\right)$ was already introduced in Section 1 . Under the assumption that the diffusion terms, determined by $\tilde{D}(r, \mu, t)$ and $\sigma^{\perp}((r, \mu, t)$, have bounded continuous partial derivatives up to order 3 and the drift $F$ has bounded first-order partial derivatives, we obtain smooth solutions, i.e., solutions which are in $\mathbf{H}_{0} \cap L_{1}\left(\mathbf{R}^{d} ; d r\right)$, provided the initial conditions are in $\mathbf{H}_{0} \cap L_{1}\left(\mathbf{R}^{d} ; d r\right)$. It follows from the duality relation of weak solutions that these densities are themselves solutions of (4.2). Under an additional smoothness and Lipschitz assumption on the coefficients we also obtain uniqueness. Further, under more smoothness assumptions on the coefficients and the initial conditions, we obtain solutions with values in the classical Hilbert-Sobolev spaces over $\mathbf{R}^{d}$, including Sobolev spaces with weights. We refer to Kotelenez 21 and Kurtz and Xiong (loc. cit.) for the general case and to Kotelenez [18] for the case where $\sigma^{\perp} \equiv 0$. Let us now just assume there is a smooth solution $X(\cdot)$ in $\mathbf{H}_{0}$ which is in the domain of the operator

$$
\left.\begin{array}{c}
\bar{A}(t, X):=A(t, X)+\tilde{A}(t, X), \quad \text { where } \\
A(t, X):=\frac{1}{2} \sum_{k, \ell=1}^{d} \partial_{k \ell, r}^{2}\left(D_{k \ell}(\cdot, X, t) X\right), \\
\tilde{A}(t, X):=\frac{1}{2} \sum_{k, \ell=1}^{d} \partial_{k \ell, r}^{2}\left(\left(\left(\sigma^{\perp}(\cdot, X, t)\right)^{2}\right)_{k \ell} X\right) .
\end{array}\right\}
$$

\footnotetext{
${ }^{14}$ Cf. (3.5).
} 
Similarly to the classical variational approach by Pardoux 31 and Krylov and Rozovski [25] we can derive an Itô formula for the square of the $L_{2}$-norm (Kotelenez [18, 21]):

$$
\left.\begin{array}{rl}
|X(t)|_{0}^{2} & =|X(0)|_{0}^{2} \\
& +\int_{0}^{t}\left\{\langle 2 \tilde{A}(s, X(s)), X(s)\rangle_{0}-\frac{1}{2} \sum_{k, \ell=1}^{d} \int_{0}^{t} \int|X(s)|_{0}^{2}\left(\partial_{k \ell}^{2} \tilde{D}\right)_{k \ell}(0, s) d s\right. \\
& +\sum_{\ell=1}^{d} \int_{0}^{t} \int X^{2}(s, r) \partial_{\ell} F_{\ell}(r, X, s) d r d s \\
& \left.+\iint_{0}^{t} X^{2}(s, r) \int \nabla \bullet(\mathcal{J}(r, p, \mathcal{X}, t) w(d p, d s))\right) d r,
\end{array}\right\}
$$

where, as before, the partial derivatives are taken with respect to $r$. Following the terminology of Krylov and Rozovski (loc. cit.), we see that the pair consisting of the sum of the second-order drift operators and the first-order diffusion operators in (4.2) is coercive if $\sigma^{\perp}$ is invertible. In what follows we will call an SPDE "coercive" if the pair is coercive. Otherwise the SPDE will be called "noncoercive". So, we also see that (4.3) is noncoercive if

$$
\sigma^{\perp} \equiv 0,
$$

which is equivalent to saying that (1.13) is noncoercive.

REMARK 4.2. Under the conditions of Theorem 4.1 we obtain the flow representation of the solution of (4.2),

$$
\mathcal{X}(t)=\int \delta_{(r(t, \mathcal{X}, 0, q))} \mathcal{X}(0, d q)=\mathcal{X}(0)\left(r^{-1}(t, \mathcal{X}, q, 0)\right),
$$

where $r(\cdot, \mathcal{X}, q, 0)$ is the solution of $(2.5)$ with input process $\tilde{\mathcal{Y}}(\cdot):=\mathcal{X}(\cdot)$ and start $q$ at 0 .

REMARK 4.3. Consider the SPDE's (4.2) and suppose that both $\mathcal{J}$ and $\sigma^{\perp}$ do not depend on the measure variable in addition to the conditions of Theorem 4.1. Further suppose that $\sigma^{\perp}$ has bounded continuous second-order partial derivatives and suppose (5.5) and (5.7) from the following Section 5. Let $\mathcal{X}(\cdot):=\mathcal{X}\left(\cdot, \mathcal{X}_{0}\right)$ be the Itô solution of the SPDE (4.2). Then $\mathcal{X}(\cdot)$ is a weak solution of the following SPDE in Stratonovich form:

$$
\begin{gathered}
d \mathcal{X}=\frac{1}{2} \sum_{k, \ell=1}^{d} \partial_{k \ell, r}^{2}\left(\left(\left(\sigma^{\perp}(\cdot, t)\right)^{2}\right)_{k \ell} \mathcal{X}\right) \\
-\nabla \bullet(\mathcal{X} F(\cdot, \mathcal{X}, t)) d t-\nabla \bullet( \\
\mathcal{X}(0)=\mathcal{X}_{0},
\end{gathered}
$$

where, as before, "o" denotes Stratonovich differentials.

Proof. By the same argument as for (1.21) we obtain a first-order SPDE contribution from the noncoercive part and the assumptions imply that the tagged noise term disappears in the limit.

5. Appendix: Stratonovich differentials. Let $\tilde{a}(\cdot)=\tilde{b}(\cdot)+\tilde{m}(\cdot)$ and $a(\cdot)=b(\cdot)+$ $m(\cdot)$ be continuous locally square integrable real-valued semi-martingales, adapted to the filtration $\mathcal{F}_{t}$, such that $\tilde{b}(\cdot)$ and $b(\cdot)$ are processes of bounded variation and $\tilde{m}(\cdot)$ and $m(\cdot)$ are martingales. Further, let $\left\{t_{0}^{n}<t_{1}^{n}<\cdots<t_{k}^{n}<\cdots\right\}$ be a sequence of partitions 
of $[0, \infty)$ whose mesh tends to zero, as $n \rightarrow \infty$. Set

$$
S_{n}(t, \tilde{a}, a):=\sum_{k} \frac{1}{2}\left\{\tilde{a}\left(t_{k}^{n}\right)+\tilde{a}\left(t_{k-1}^{n}\right)\right\}\left(a\left(t_{k}^{n}\right)-a\left(t_{k-1}^{n}\right)\right) .
$$

Ikeda and Watanabe show that $S_{n}(t, \tilde{a}, a)$ converges in probability, uniformly on compact intervals $[0, T]$. The proof of this statement is very similar to the proof of the Itô formula. Thus, we obtain the "Stratonovich integral of $\tilde{a}(\cdot)$ with respect to a $(d s)$ ":

$$
\int_{0}^{t} \tilde{a}(s) \circ a(d s):=\lim _{n \rightarrow \infty} S_{n}(t, \tilde{a}, a) .
$$

The representation of the approximating sequence immediately implies the following transformation rule:

$$
\int_{0}^{t} \tilde{a}(s) \circ a(d s) \equiv \int_{0}^{t} \tilde{a}(s) a(d s)+[\tilde{m}, m](t) \text { a.s. }
$$

where the stochastic integral on the right-hand side is the Itô integral. The mutual quadratic variation $[\tilde{m}, m](\cdot)$ in $(5.2)$ is called the "correction term", which we have to add to the Itô integral to obtain the corresponding Stratonovich integral. The generalization to multidimensional semi-martingales follows from the real case componentwise. Let us recall the chain rule. Suppose $a(\cdot):=\left(a_{1}(\cdot), \ldots, a_{d}(\cdot)\right)$ is an $\mathbf{R}^{d}$-valued continuous locally square integrable semi-martingale and $\varphi \in C^{3}\left(\mathbf{R}^{d} ; \mathbf{R}\right)$. Then,

$$
\tilde{a}(\cdot):=\varphi(a(\cdot))
$$

is a real-valued continuous square integrable semi-martingale and the following representation holds:

$$
\tilde{a}(t) \equiv \varphi(a(0))+\sum_{i=1}^{d} \int_{0}^{t}\left(\frac{\partial}{\partial r_{i}} \varphi\right)(a(s)) \circ a_{i}(d s) .
$$

In what follows we will apply the Stratonovich integral to the study of a special case of semi-martingales, given by the solutions of (1.19), where for notational convenience we replace $\mathcal{X}_{\varepsilon, N}$ by an adapted continuous measure-valued process $\tilde{\mathcal{Y}}(\cdot)$ and drop the subscript " $\varepsilon$ " at the coefficients and solutions. More precisely, we consider

$$
\left.\begin{array}{l}
d r(t)=F(r(t), \tilde{\mathcal{Y}}(t), t) d t+\int \mathcal{J}(r(t), p, t) w(d p, d t), \\
r(0)=r_{0},
\end{array}\right\}
$$

assuming the conditions of (2.4) and (4.5). Next, let $z(\cdot)$ be a continuous square integrable $\mathbf{R}^{d}$-valued semi-martingale. Further, suppose that, in addition to $(2.4), \mathcal{J}(r, q, s)$ is twice continuously differentiable with respect to $r$ such that

$$
\sup _{r \in \mathbf{R}^{d}} \sum_{i, j, k, \ell=1}^{d} \int_{0}^{T} \int\left(\frac{\partial^{2}}{\partial r_{i} \partial r_{j}} \mathcal{J}_{k, \ell}\right)^{2}(r, q, s) d q d s<\infty .
$$

Employing the representation (1.16)/(1.17) and applying Itô's formula to

$$
\varphi(r, t):=\sigma_{n, k \ell}(r, \mu, t), \quad k, \ell=1, \ldots, d,
$$

we obtain that all $n$ entries of $\sigma_{n, k \ell}(z(\cdot), \mu, \cdot)$ are continuous square integrable $\mathbf{R}^{d}$-valued semi-martingales and the same holds for the sum. Consequently, we can define the 
Stratonovich integral of $\mathcal{J}(z(s), q, s)$ with respect to $w(d q, d s)$ as a series of Stratonovich integrals

$$
\int_{0}^{t} \int \mathcal{J}(z(s), q, s) w(d q, \circ d s):=\sum_{n=1}^{\infty} \int_{0}^{t} \sigma_{n}(z(s), s) \circ \beta^{n}(d s) .
$$

Proposition 5.1. Let $r(\cdot)$ be the solution of (5.4). In addition to (2.4) and (5.5) suppose that the diffusion matrix $\tilde{D}_{k \ell}$, associated with the diffusion kernel $\mathcal{J}_{k, \ell}(r, q, s)$, is spatially homogeneous and that the divergence of the diffusion matrix equals 0 at $(0, t) \forall t$, i.e.,

$$
\sum_{k=1}^{d}\left(\partial_{k} \tilde{D}\right)_{k \ell}(0, t) \equiv 0 \quad \forall \ell
$$

Then

$$
\int_{0}^{t} \int \mathcal{J}(r(s), q, s) w(d q, \circ d s)=\int_{0}^{t} \int \mathcal{J}(r(s), q, s) w(d q, d s)
$$

i.e., the Stratonovich and the Itô integrals coincide in this particular case.

Proof. The solution of (5.4), $r(\cdot)$, is obviously a continuous square integrable $\mathbf{R}^{d_{-}}$ valued semi-martingale. Itô's formula provides the representation of $\sigma_{n, i j}(r(\cdot), \cdot)$ as continuous square integrable semi-martingales. Hence, the correction term

$$
\left[\sigma_{n, i j}(r(t), t), \int_{0}^{t} \beta_{j}^{n}(d s)\right]
$$

satisfies

$$
\left.\begin{array}{l}
{\left[\sigma_{n, i j}(r(t), t), \int_{0}^{t} \beta_{j}^{n}(d s)\right] \equiv \sum_{k=1}^{d}\left[\int_{0}^{t}\left(\partial_{k} \sigma_{n, i j}\right)(r(s), s) r_{k}(d s), \int_{0}^{t} \beta_{j}^{n}(d s)\right]} \\
\equiv \sum_{k=1}^{d}\left[\int_{0}^{t} \int\left(\partial_{k} \sigma_{n, i j}\right)(r(s), s)\left\{\sum_{m} \sum_{\ell=1}^{d} \sigma_{m, k \ell}(r(s), s) \beta_{\ell}^{m}(d s)\right\}, \int_{0}^{t} \beta_{j}^{n}(d s)\right] \\
\equiv \sum_{k=1}^{d} \int_{0}^{t} \int\left(\partial_{k} \sigma_{n, i j}\right)(r(s), s) \sigma_{n, k j}(r(s), s) d s,
\end{array}\right\}
$$

where the last line follows from the independence of $\beta_{j}^{n}$ and $\beta_{\ell}^{m}$ if $(n, j) \neq(m, \ell)$. Summing up the correction terms and using a more traditional notation for partial derivatives, we obtain for the correction term,

$$
\left.\sum_{k=1}^{d}\left(\partial_{k} \sum_{n} \sum_{j=1}^{d} \sigma_{n, i j}(r, t) \sigma_{n, k j}(q, t)_{\mid q=r}\right)=\sum_{k=1}^{d}\left(\frac{\partial}{\partial r_{k}} \int(\mathcal{J}(r, p, t) \mathcal{J}(q, p, t))_{i k} d p\right)_{\mid q=r}\right) .
$$

However,

$$
\int(\mathcal{J}(r, p, t) \mathcal{J}(q, p, t))_{i k} d p=\tilde{D}_{i k}(r-q, t)
$$

Hence,

$$
\left.\sum_{k=1}^{d} \sum_{n} \sum_{j=1}^{d}\left(\partial_{k} \sigma_{n, i j}\right)(r, t)\right) \sigma_{n, k j}(q, t)_{\mid q=r} \equiv \sum_{k=1}^{d}\left(\partial_{k} \tilde{D}\right)_{i k}(r-q, t)_{\mid q=r} \equiv 0,
$$

by assumption (5.7). We conclude that the sum of all correction terms equals 0 , which implies (5.8). 
Lemma 5.2. Let $\varphi \in C^{3}\left(\mathbf{R}^{d} ; \mathbf{R}\right)$ and assume the conditions and notation of Proposition 5.1. Then

$$
\sum_{n} \int_{0}^{t}(\nabla \varphi(r(s))) \bullet\left(\circ \sigma_{n}(r(s), s) \beta^{n}(d s)\right)=\sum_{n} \int_{0}^{t}(\nabla \varphi(r(s))) \bullet\left(\sigma_{n}(r(s), s) \circ \beta^{n}(d s)\right) .
$$

Proof. As in the proof of Proposition 5.1 we first analyze the one-dimensional coordinates of (5.11). Since the deterministic integral $\int_{0}^{t} F(r(s), \tilde{\mathcal{Y}}, s) d s$ is the same for Itô and Stratonovich integrals, we may in what follows assume, without loss of generality, $F \equiv 0$. By $(5.2)$,

$$
\left.\begin{array}{l}
\sum_{j=1}^{d} \int_{0}^{t}\left(\partial_{k} \varphi\right)(r(s)) \circ \sigma_{n, k j}(r(s), s) \beta_{j}^{n}(d s) \\
=\sum_{j=1}^{d} \int_{0}^{t}\left(\partial_{k} \varphi\right)(r(s)) \sigma_{n, k j}(r(s), s) \beta_{j}^{n}(d s) \\
\quad+\frac{1}{2}\left[\left(\partial_{k} \varphi\right)(r(t)), \sum_{j=1}^{d} \int_{0}^{t} \sigma_{n, k j}(r(s), s) \beta_{j}^{n}(d s)\right] .
\end{array}\right\}
$$

As before, we employ Itô's formula to obtain the martingale part of $\frac{\partial}{\partial r_{k}} \varphi(r(t))$. Then, as in (5.9),

$$
\begin{aligned}
\frac{1}{2}\left[\left(\partial_{k} \varphi\right)(r(t)), \sum_{j=1}^{d} \int_{0}^{t} \sigma_{n, k j}(r(s), s) \beta_{j}^{n}(d s)\right] \\
=\frac{1}{2} \sum_{j, \ell=1}^{d} \int_{0}^{t}\left(\partial_{k \ell}^{2} \varphi\right)(r(s)) \sigma_{n, \ell j}(r(s), s) \sigma_{n, k j}(r(s), s) d s .
\end{aligned}
$$

Summing up over all $n$ yields for the left-hand side of (5.11):

$$
\frac{1}{2}\left[\left(\partial_{k} \varphi\right)(r(t)), \sum_{n} \sum_{j=1}^{d} \int_{0}^{t} \sigma_{n, k j}(r(s), s) \beta_{j}^{n}(d s)\right]=\frac{1}{2} \sum_{\ell=1}^{d} \int_{0}^{t}\left(\partial_{k \ell}^{2} \varphi\right)(r(s)) D_{\ell k}(0, s) d s .
$$

For the right-hand side of (5.11) we have

$$
\left.\begin{array}{l}
\sum_{j=1}^{d} \int_{0}^{t}\left(\partial_{k} \varphi\right)(r(s)) \sigma_{n, k j}(r(s), s) \circ \beta_{j}^{n}(d s) \\
=\sum_{j=1}^{d} \int_{0}^{t}\left(\partial_{k} \varphi\right)(r(s)) \sigma_{n, k j}(r(s), s) \beta_{j}^{n}(d s) \\
\quad+\frac{1}{2} \sum_{j=1}^{d}\left[\left(\partial_{k} \varphi\right)(r(t)) \sigma_{n, k j}(r(t), t), \int_{0}^{t} \beta_{j}^{n}(d s)\right] .
\end{array}\right\}
$$

By Itô's formula, the martingale term of $\left(\partial_{k} \varphi\right)(r(t)) \sigma_{n, k j}(r(t), t)$ equals

$$
\begin{aligned}
\int_{0}^{t} \sigma_{n, k j}(r(s), s)\left(\nabla \partial_{k} \varphi\right) & (r(s)) \bullet \int \mathcal{J}(r(s), p, s) w(d p, d s) \\
+ & \int_{0}^{t}\left(\partial_{k} \varphi\right)(r(s))\left(\nabla \sigma_{n, k j}(r(s), s)\right) \bullet \int \mathcal{J}(r(s), p, s) w(d p, d s),
\end{aligned}
$$

whence the correction term in (5.15) satisfies the following equation:

$$
\left.\begin{array}{l}
\frac{1}{2} \sum_{j=1}^{d}\left[\left(\partial_{k} \varphi\right)(r(t)) \sigma_{n, k j}(r(t), t), \int_{0}^{t} \beta_{j}^{n}(d s)\right] \\
=\frac{1}{2} \sum_{\ell, j=1}^{d} \int_{0}^{t}\left(\partial_{k \ell}^{2} \varphi\right)(r(s)) \sigma_{n, k j}(r(s), s) \sigma_{n, \ell j}(r(s), s) d s \\
\left.+\frac{1}{2} \sum_{\ell, j=1}^{d} \int_{0}^{t}\left(\partial_{k} \varphi\right)(r(s))\left(\partial_{\ell} \sigma_{n, k j}\right)(r(s), s)\right) \sigma_{n, \ell j}(r(s), s) d s .
\end{array}\right\}
$$


The sum of the second terms over all $n$ equals 0 by (5.10). Summing up the first terms over all $n$ in the right-hand side of (5.16) yields

$$
\left.\begin{array}{l}
\frac{1}{2} \sum_{\ell, j=1}^{d} \int_{0}^{t}\left(\partial_{k \ell}^{2} \varphi\right)(r(s)) \sum_{n} \sigma_{n, k j}(r(s), s) \sigma_{n, \ell j}(r(s), s) d s \\
=\frac{1}{2} \sum_{\ell=1}^{d} \int_{0}^{t}\left(\partial_{k \ell}^{2} \varphi\right)(r(s)) D_{k \ell}(0, s) d s .
\end{array}\right\}
$$

Since the diffusion matrix $D_{k \ell}$ is symmetric, we obtain that the correction terms for both sides of (5.11) are equal.

We can now make the following important observation:

Theorem 5.3. Suppose that the conditions of Proposition 5.1 hold and let $\varphi \in$ $C^{3}\left(\mathbf{R}^{d} ; \mathbf{R}\right)$. Denoting by $r(\cdot)$, the solution of the (Itô) SODE (5.4), the following holds:

$$
\left.\begin{array}{l}
\varphi(r(t)) \equiv \frac{1}{2} \sum_{k, \ell=1}^{d}\left(\partial_{k \ell}^{2} \varphi\right)(r(s)) D_{k \ell}(0, s) d s \\
+\sum_{k=1}^{d} \int_{0}^{t}\left(\nabla \varphi(r(s)) \bullet \int \mathcal{J}_{k, \ell}(r(s), q, s) w_{\ell}(d q, d s)+\int_{0}^{t}(\nabla \varphi)(r(s)) \bullet F(r(s), \tilde{\mathcal{Y}}(s), s) d s\right. \\
=\sum_{k=1}^{d} \int_{0}^{t}\left(\nabla \varphi(r(s)) \bullet \int \mathcal{J}_{k, \ell}(r(s), q, s) w_{\ell}(d q, \circ d s)+\int_{0}^{t}(\nabla \varphi)(r(s)) \bullet F(r(s), \tilde{\mathcal{Y}}(s), s) d s .\right.
\end{array}\right\}
$$

Proof. By assumption (4.5), the diffusion matrix $D(\cdot, t) \equiv D(0, t)$. Hence, the Itô formula yields the the first part of (5.18). The chain rule for Stratonovich integrals in addition to Lemma 5.2 implies that $\varphi(r(t))$ equals the right-hand side of (5.18).

\section{REFERENCES}

[1] Borkar, V.S., Evolution of Interacting Particles in a Brownian Medium. Stochastics 14 (1984), 33-79. MR774584 (86f:60073)

[2] Dawson, D.A., Vaillancourt, J., and Wang, H., Stochastic Partial Differential Equations for a Class of Interacting Measure-valued Diffusions. Ann. Inst Henri Poincaré, Probabilités et Statistiques 36 (2000), no. 2, 167-180. MR.1751657(2002d:60053)

[3] Dorogovtsev, A., One Brownian Stochastic Flow. Theory of Stochastic Processes, 10 (2004), no. 3-4, 21-25. MR.2329772

[4] Dürr, D., Goldstein, S. and Lebowitz, J.L., A Mechanical Model of Brownian Motion. Commun. Math. Phys. 78 (1981), 507-530. MR606461 (83d:60109)

[5] Dynkin, E.B., Markov processes. Vol. I. Springer-Verlag, Berlin-Göttingen-Heidelberg, 1965.

[6] Einstein, A., Über die von der molekularkinetischen Theorie der Wärme gefordete Bewegung von in ruhenden Flüssigkeiten suspendierten Teilchen. Ann.d.Phys. 17 (quoted from the English translation: (1956) Investigations on the Theory of Brownian Movement, Dover Publications, Inc., New York. MR0077443 (17:1035g)

[7] Ethier, S.N. and Kurtz, T.G., Markov Processes. Characterization and Convergence. John Wiley \& Sons, New York - Toronto, 1986. MR838085 (88a:60130)

[8] Friedman, A., Stochastic differential equations and applications. Vol. 2, Academic Press. New York-San Francisco-London, 1976. MR0494491 (58:13350b)

[9] Gärtner, J.: On the McKean-Vlasov limit for interacting diffusions. Math. Nachr. 137 (1988), 197248. MR968996 (90a:60184)

[10] Gel'fand, I.M. and Vilenkin, N. Ya., Generalized Functions. Vol. 4. Academic Press. New York London, 1964. MR0435834 (55:8786d)

[11] Gikhman, I.I. and Skorokhod, A.V., Stochastic differential equations. Naukova Dumka, Kiev (in Russian - English Translation (1972): Stochastic Differential Equations. Springer-Verlag, Berlin).

[12] Goncharuk, N. and Kotelenez, P., Fractional Step Method for Stochastic Evolution Equations. Stoch. Proc. Appl. 73 (1998), 1-45. MR1603842 (99b:60090)

[13] Haken, H., Advanced Synergetics. Springer-Verlag, Berlin-Heidelberg, New York, Tokyo, 1983. MR707096 (86h:00020)

[14] Ikeda, N. and Watanabe, S., Stochastic differential equations and diffusion processes. NorthHolland Publishing Company. Amsterdam - New York, 1989. MR.1011252 (90m:60069) 
[15] Jetschke, G., On the Equivalence of Different Approaches to Stochastic Partial Differential Equations. Math. Nachr. 128 (1986), 315-329. MR855965 (87k:60156)

[16] Kampen, N.G. van, Stochastic Processes in Physics and Chemistry. North-Holland Publ. Co., Amsterdam, New York, 1983.

[17] Kotelenez, P., A Stochastic Navier-Stokes Equation for the Vorticity of a Two-dimensional Fluid. Ann. Applied Probab. 5 (1995), No. 4, 1126-1160. MR1384369 (97f:60116)

[18] Kotelenez, P., A Class of Quasilinear Stochastic Partial Differential Equations of McKean-Vlasov Type with Mass Conservation. Probab. Theory Relat. Fields. 102 (1995), 159-188 MR1337250 (96k:60157)

[19] Kotelenez, P., From Discrete Deterministic Dynamics to Stochastic Kinematics - A Derivation of Brownian Motions. Stochastics and Dynamics 5 (2005), Number 3, 343-384. MR2166985

[20] Kotelenez, P., Correlated Brownian Motions as an Approximation to Deterministic Mean-Field Dynamics. Ukrainian Math. J. 57 (2005), no. 6, 757-769 MR.2208453(2006k:82125)

[21] Kotelenez, P., Stochastic Ordinary and Stochastic Partial Differential Equations - Transition from Microscopic to Macroscopic Equations. Springer-Verlag, Berlin-Heidelberg-New York, 2007.

[22] Kotelenez, P., Leitman M. and Mann, J. Adin Jr., On the Depletion Effect in Colloids. Preprint.

[23] Kotelenez, P. and Kurtz, T.G., Macroscopic Limit for Stochastic Partial Differential Equations of McKean-Vlasov Type. (Preprint)

[24] Krylov, N.V., Private Communication.

[25] Krylov, N.V. and Rozovsky, B.L., On stochastic evolution equations. Itogi Nauki i tehniki, VINITI, 71-146, 1979 (in Russian).

[26] Kunita, H., Stochastic Flows and Stochastic Differential Equations. Cambridge University Press, Cambridge, New York, Port Chester, Melbourne, Sydney, 1990. MR1472487 (98e:60096)

[27] Kurtz, T.G. and Xiong, Jie, Particle Representations for a Class of Nonlinear SPDEs. Stochastic Process Appl. 83 (1999), 103-126. MR.1705602 (2000g:60108)

[28] Lifshits, E.M. and Pitayevskii, L.P., Physical Kinetics. Theoretical Physics X. Nauka, Moscow, 1979 (in Russian).

[29] Metivier, M. and Pellaumail, J., Stochastic Integration. Adademic Press, New York, 1980.

[30] Oelschläger, K., A Martingale Approach to the Law of Large Numbers for Weakly Interacting Stochastic Processes. Ann. Probab. 12 (1984), 458-479.

[31] Pardoux, E., Stochastic Partial Differential Equations and Filtering of Diffusion Processes. Stochastics Vol. 3, 1979, 127-167. MR553909 (81b:60059)

[32] Rozovsky, B.L., Stochastic Evolution Systems. Nauka, Moscow (in Russian - English Translation (1990), Kluwer Academic, Dordrecht-Boston). MR.1135324 (92k:60136)

[33] Sinai, Ya. G. and Soloveichik, M.R., One-Dimensional Classical Massive Particle in the Ideal Gas. Commun. Math. Phys. 104 (1986), 423-443. MR840745 (88h:82014)

[34] Szász, D. and Tóth, B., Towards a Unified Dynamical Theory of the Brownian Particle in an Ideal Gas. Commun. Math. Phys. 111 (1986), 41-62. MR896758(89d:82021)

[35] Truesdell, C. and Noll, W., Encyclopedia of Physics - Volume III/3 - The Nonlinear Field Theories of Mechanics. Springer-Verlag, Berlin-Heidelberg-New York, 1965. MR.1215940 (94c:73002)

[36] Vaillancourt, J., On the Existence of Random McKean-Vlasov limits for Triangular Arrays of Exchangeable Diffusions. Stoch. Anal. Appl. 6(4) (1988), 431-446. MR964251(90a:60142)

[37] Walsh, J.B., An Introduction to Stochastic Partial Differential Equations. Ecole d'Eté de Probabilité de Saint Fleur XIV. Lecture Notes in Math. 1180. Springer, Berlin, 1986, pp. 265-439. MR 876085 (88a:60114)

[38] Wong, E. and Zakai, M., On the Relation between Ordinary and Stochastic Differential Equations, Int. J. Eng. Sci. 3 (1965), 213-229. MR0183023(32:505) 